\title{
IncRNA PART1, manipulated by transcriptional factor FOXP2, suppresses proliferation and invasion in ESCC by regulating the miR-18a-5p/SOX6 signaling axis
}

\author{
YAN ZHAO $^{1}$, QING ZHANG $^{2}$, HONGTAO LIU $^{2}$, NING WANG $^{3}$, XIAOSAN ZHANG $^{1}$ and SHUJUN YANG ${ }^{1}$ \\ ${ }^{1}$ Department of Medical Oncology, Affiliated Cancer Hospital of Zhengzhou University, Zhengzhou, Henan 450008; \\ ${ }^{2}$ School of Life Sciences, Zhengzhou University, Zhengzhou, Henan 450001; ${ }^{3}$ Henan Academy of Medical and \\ Pharmaceutical Sciences, Zhengzhou University, Zhengzhou, Henan 450052, P.R. China
}

Received August 14, 2020; Accepted November 24, 2020

DOI: $10.3892 /$ or.2021.7931

\begin{abstract}
An increasing number of studies have demonstrated that long non-coding (lnc)RNAs are associated with tumor invasion, metastasis and the prognosis of patients with a variety of different tumors. However, the roles of IncRNA prostate androgen regulated transcript 1 (PART1) in esophageal squamous cell carcinoma (ESCC) remain unknown. In the present study, reverse transcription-quantitative PCR was performed to investigate the levels of PART1, SRY-box transcription factor 6 (SOX6) and miR-18a-5p in ESCC tissues and cells. The functions of PART1 in ESCC were demonstrated using Cell Counting Kit- 8 and Matrigel assays. Promoter activity and dual-luciferase reporter assays, RNA immunoprecipitation and western blot analyses were also used to determine the potential mechanisms of PART1 in ESCC cell lines. It was found that PART1 and SOX6 were both downregulated in ESCC tissues and cells, and their low expression levels were associated with TNM stage, lymph node metastasis and poor prognosis in patients with ESCC. Forkhead box protein P2 (FOXP2) exhibited low expression level in ESCC tissues, and its expression was positively correlated with PART1 expression level in ESCC tissues. FOXP2 was found to bind to the promoter region of PART1 to regulate its expression in ESCC cells. Functionally, PART1 overexpression suppressed cell proliferation and invasion, whereas PART1 downregulation promoted cell proliferation and invasion in the ESCC cell lines. Mechanistically, PART1 functions as a competing endogenous (ce)RNA by sponging miR-18a-5p, resulting in the
\end{abstract}

Correspondence to: Professor Shujun Yang, Department of Medical Oncology, Affiliated Cancer Hospital of Zhengzhou University, 127 Dongming Road, Zhengzhou, Henan 450008, P.R. China

E-mail: nkyang001@126.com

Key words: esophageal squamous cell carcinoma, long non-coding RNA, prostate androgen regulated transcript 1, microRNA-18a-5p, SOX6 upregulation of the downstream target gene, SOX6, coupled with the inactivation of the $\beta$-catenin/c-myc signaling axis, to suppress ESCC cell proliferation and invasion. In conclusion, data from the present study unveil a potential ceRNA regulatory pathway, in which PART1 affects SOX6 expression level by sponging miR-18a-5p, to ultimately suppress ESCC development and progression.

\section{Introduction}

Esophageal cancer (ESCA), as a complex disease harboring numerous pathogenic factors, is the sixth leading cause of cancer-associated death worldwide (1). There are two main histological types, including esophageal squamous cell carcinoma (ESCC) and esophageal adenocarcinoma, which exhibit completely distinct geographical distributions and primary risk factors (2). Patients with ESCC account for $>90 \%$ of all patients with ESCA in China (3). The prognosis of patients with ESCA remains poor, with a 5-year survival rate of only $25 \%$ (4), which is primarily due to the late-stage at diagnosis and distant metastasis. Thus, there is an urgent requirement to identify novel prognostic markers and new molecular targets to improve the prognosis and therapeutic efficacy in patients with ESCC.

Long non-coding ( $\operatorname{lnc})$ RNAs are considered to be a novel class of transcripts, that are $>200$ nucleotides in length, do not have any protein-coding potential (5), and are typically transcribed by RNA polymerase II, which mirrors that of protein-coding genes (6). Currently, lncRNAs consist of five different molecular types according to their genomic location on the chromosome, including sense, antisense, intronic, intergenic and bidirectional lncRNAs (7-9). IncRNAs have been associated with the regulation of numerous essential biological processes, such as allosterically manipulating enzymatic activity, shaping chromosome conformation, functioning as regulators of histone methylation and imprinting genomic loci (9-11). Recently, lncRNAs have been associated with tumor initiation, progression and metastasis $(12,13)$, and play important roles in the survival, growth, differentiation, apoptosis and regulating signal pathways in a number of tumors (14-18). Notably, IncRNAs have been found to be novel prognostic markers and therapeutic targets for numerous types 
of cancer $(19,20)$. Prostate androgen regulated transcript 1 (PART1) is predominately expressed in the prostate and is transcriptionally regulated by androgens in prostate cancer cells (21). At present, PART1, as a novel lncRNA, has been associated with tumor progression, metastasis and chemotherapy resistance $(22,23)$. In addition, PART1 has been found to be involved in the regulation of several signaling pathways, such as the PTEN/AKT signaling pathway (24), the microRNA (miR)-150-5p/LRG1 axis (22) and the miR-429/SHCBP1 regulatory network (25), suggesting that targeting PART1 may be a novel and promising therapeutic strategy in patients with cancer. Therefore, elucidation of the function of PART1 and its molecular mechanisms, may provide further understanding and enable treatment in a clinical setting for different types of cancer. However, to date, the functions and molecular mechanisms of PART1 in ESCC remain unclear.

Recently, forkhead box protein P2 (FOXP2) has been verified to be a tumor suppressor (26). Notably, FOXP2 suppresses the transcriptional activity of numerous downstream target genes, via its zinc finger domain, further affecting tumor progression $(26,27)$. In addition, FOXP2 was found to be essential for the suppression of cell growth by regulating p21 in osteosarcoma cells (28). These findings suggest that FOXP2 may function as a key regulator in various types of cancer by regulating its target genes.

In the present study, the expression level of PART1 in ESCC tissues and cells was investigated, and the clinical value of PART1 in TNM stage, lymph node metastasis and the prognosis of patients with ESCC was also determined. In addition, FOXP2 was found to be a transcription factor, that regulated the expression level of PART1 by binding to its promoter region and acted as a competing endogenous (ce)RNA by sponging miR-18a-5p to promote the expression level of SRY-box transcription factor 6 (SOX6), which further triggered the inactivation of the $\beta$-catenin/c-myc signal axis, resulting in the suppression of cell proliferation and invasion in ESCC. Collectively, the PART1/miR-18a-5p/SOX6/ $\beta$-catenin signaling axis uncovered in the present study may be a novel therapeutic target for patients with ESCC.

\section{Materials and methods}

Bioinformatics analysis. The GEO GSE111011 dataset (https://www.ncbi.nlm.nih.gov/gds/?term=GSE111011) was downloaded to analyze the levels of PART1 and SOX6 in patients with ESCC, from tumor and paired normal samples. The GEO GSE43732 (29) was used to investigate the miR-18a-5p expression in ESCC samples. The StarBase online database v2.0 (30) was also used to investigate the levels of PART1 and miR-18a-5p in patients with ESCA and in normal samples. Correlation analysis between PART1 and SOX6, as well as the prognostic value of SOX6 was also examined using the StarBase database. The upstream promoter sequence of PART1 was detected using the promoter analysis tools, UCSC (http://genome.ucsc. edu/) and JASPAR 2020 (http://jaspar.genereg.net/), while the lncATLAS online software (http://lncatlas.crg.eu/) was used to predict the subcellular localization of PART1. Targetscan and miRDB online software programs were used to predict the possible binding sites of miR-18a-5p in the SOX6 3'-untranslated region (UTR).
Tissue samples. A total of 75 ESCC samples and paired adjacent normal tissues were obtained from the Affiliated Cancer Hospital of Zhengzhou University (Henan, China) from 2015 to 2019 , including 49 males and 26 females; 40 cases $\geq 60$ years of age, and 35 cases $<60$ years. Tissue samples were confirmed using hematoxylin and eosin staining by experienced pathologists from the Affiliated Cancer Hospital of Zhengzhou University (Henan, China). Informed consent was provided by each patient and the utilization of all the samples was approved by the Research and Ethics Committee of the Affiliated Cancer Hospital of Zhengzhou University (Henan, China) (approval no. 2019016).

Cell lines and cell culture. The human ESCC cell lines (Eca109, EC9706, TE1, KYSE70 and KYSE450) and the normal Het-1A esophageal epithelial cell line were purchased from the Chinese Academy of Sciences Cell Bank, and were cultured in RMPI-1640 medium, supplemented with $10 \%$ fetal bovine serum (FBS) (Gibco; Invitrogen; Thermo Fisher Scientific, Inc.) in a humidified incubator with $5 \% \mathrm{CO}_{2}$.

Cell transfection. Small interfering (si)RNAs (50 $\mathrm{nM})$ (Table I), miR-18a-5p mimic (50 $\mathrm{nM}$, forward (F), 5'-UAAGGUGCAUCUAGUGCAGAUAG-3' and reverse (R), 5'-AUCUGCACUAGAUGCACCUUAUU-3'); negative control (NC, F, 5'-UUCUCCGA ACGUGUCACGUTT-3' and R, 5'-ACGUGACACGUUCGGAGAATT-3'); miR-18a-5p inhibitor (50 nM, 5'-CUAUCUGACUAGAUGCACCUUA-3'); NC inhibitor (50 nM, 5'-UUCUCCGAACGUGUCACGUTT-3') (Shanghai GenePharma, Co., Ltd.), pcDNA3.1 (2.5 $\mu \mathrm{g})$, and pcDNA3.1-PART1/FOXP2/SOX6 (2.5 $\mu \mathrm{g}$ ) (TsingKe Biological Technology) were transfected into the Eca109 and EC9706 cell lines when cells reached approximately $80 \%$ confluence using Lipofectamine $^{\mathrm{TM}} 2000$ (Invitrogen; Thermo Fisher Scientific, Inc.) at room temperature for about $20 \mathrm{~min}$ according to the manufacturer's instructions. The subsequent experiment was performed $48 \mathrm{~h}$ after transfection.

Cell counting Kit-8 (CCK-8) assay. Cell proliferation of the ESCC cell lines, with various treatments was performed in triplicate according to the manufacturer's protocol, then the ESCC cells $(\sim 2,000$ cells/well) were seeded into a 96-well plate. At the time of measurement, $\mathrm{CCK}-8$ reagent (Beyotime Institute of Biotechnology) was added to the corresponding wells, and the absorbance (450 nm) was measured using a microplate reader (Thermo Fisher Scientific, Inc.).

Matrigel assay. Cell invasion was investigated using a Transwell chamber harboring Matrigel (BD Biosciences). Briefly, the Eca109 and EC9706 cell lines (1x10 $)$ were added to the upper layer of the chamber and $20 \%$ FBS was added to the lower chamber. Afterwards, the invasive cells were fixed with methanol, followed by staining with $0.1 \%$ crystal violet at room temperature for $15 \mathrm{~min}, 48 \mathrm{~h}$ following transfection. Finally, the number of invasive cells was determined at x200 magnification under a light microscope (Leica, Germany). The results were from three independent repeated experiments.

Reverse transcription-quantitative PCR (RT-qPCR). Total RNA was isolated using TRIzol ${ }^{\circledR}$ (Invitrogen; Thermo Fisher 
Table I. Sequences of the siRNA and si-NC used for synthesis.

\begin{tabular}{lll}
\hline siRNAs & \multicolumn{1}{c}{ Sense (5'-3') } & Antisense (5'-3') \\
\hline PART1 siRNA\#1 & GAGUUGACUUUGUGUUAUACA & UAUAACACAAAGUCAACUCUG \\
PART1 siRNA\#2 & GGUGUGAAAUAAAGGUUAAUG & UUAACCUUUAUUUCACACCUU \\
PART1 siRNA\#3 & GAAAGUUGUUGAAUAUAAACU & UUUAUAUUCAACAACUUUCAU \\
FOXP2 siRNA\#1 & CGACAGAGACAAUAAGCAACA & UUGCUUAUUGUCUCUGUCGCA \\
FOXP2 siRNA\#2 & GGCUAGACCUCACUACUAACA & UUAGUAGUGAGGUCUAGCCCU \\
FOXP2 siRNA\#3 & GCAGCAACAACAACAACAACA & UUGUUGUUGUUGUUGCUGCUG \\
SOX6 siRNA\#1 & GGAUCUCGCUGGAAAUCAAUG & UUGAUUUCCAGCGAGAUCCUA \\
SOX6 siRNA\#2 & AGAACAGAUUGCGAGACAACA & UUGUCUCGCAAUCUGUUCUUG \\
SOX6 siRNA\#3 & GAAUGGAAUCAGAGAAUAAUA & UUAUUCUCUGAUUCCAUUCUU \\
Si-NC & UUCUCCGAACGUGUCACGUTT & ACGUGACACGUUCGGAGAATT
\end{tabular}

Si, small interfering; PART1, prostate androgen regulated transcript 1; SOX6, SRY-box transcription factor 6; NC, negative control.

Scientific, Inc.) according to the manufacturer's instructions. For PART1, SOX6 and FOXP2 expression analysis, RT-qPCR was performed using the Quant One-Step RT-qPCR kit (SYBR Green, FP303) (Tiangen Biotech Co., Ltd.) and an ABI 7500 series PCR machine (Applied Biosystems; Thermo Fisher Scientific, Inc.), with the following specific primers: PART1 forward, 5'-CCAGAGCCAGCCAATCACTT-3' and reverse 5'-CTGTCCTTTTCCCCTCCGAC-3'; SOX6 forward, 5'-AAG ATGCAGAGGGAGGTGC-3' and reverse, 5'-GGTTGCTTC TCCTGGTTGGA-3'; FOXP2 forward, 5'-GCAGCCAATTAG ATGCTGGC-3' and reverse 5'-ATCATGGCCACTGACACA GG-3', GAPDH forward, 5'-CGGAGTCAACGGATTTGG TCGTAT-3'; and reverse 5'-AGCCTTCTCCATGGTGGT GAAGAC-3'). PCR reaction conditions were as follows: $95^{\circ} \mathrm{C}$ for $30 \mathrm{sec}, 30$ cycles $\left(95^{\circ} \mathrm{C}\right.$ for $5 \mathrm{sec}, 50^{\circ} \mathrm{C}$ for $30 \mathrm{sec}$, and $72^{\circ} \mathrm{C}$ for $30 \mathrm{sec}$ ). For the miR-18a-5p assay, total RNA was reverse transcribed using the miRcute Plus miRNA First-Strand cDNA kit (cat. no. KR211; Tiangen Biotech Co., Ltd.). RT-qPCR for miR-18a-5p was performed using the miRcute Plus miRNA qPCR kit (SYBR Green) (FP411; Tiangen Biotech Co., Ltd.) using the following specific forward primers, with the reverse primers from the kit: miR-18a-5p forward, 5'-TAAGGTGCA TCTAGTGCAGATAG-3'; and U6 forward, 5'-CTCGCTTCG GCAGCACA-3'). PCR reaction conditions were as follows: $95^{\circ} \mathrm{C}$ for $15 \mathrm{~min}$ in one cycle, 40 cycles $\left(95^{\circ} \mathrm{C}\right.$ for $5 \mathrm{sec}, 60^{\circ} \mathrm{C}$ for $30 \mathrm{sec}$ ). The experiments were normalized to U6. Relative gene expression was analyzed using the $2^{-\triangle \Delta C q}$ method (31). The results were from three independent repeated experiments.

Subcellular fractionation. A cell nucleus and cytoplasm RNA isolation kit (Beibei, Biotech, Co., Ltd.) was used to extract the nuclear and cytoplasmic RNA, respectively, according to the manufacturer's instructions. Then, RT-qPCR was performed to detect the levels of PART1, U6 and GAPDH. The results were from three independent repeated experiments.

Promoter activity assay. The fragments, containing wild-type (WT) or mutant (MUT) binding sites of FOXP2 in the PART1 transcript were constructed using the pGL3-basic vector (Promega Corp.). The pGL3-PART1-promoter-WT and
pGL3-PART1-promoter-MUT vectors along with pcDNA3.1 and pcDNA3.1-FOXP2 were transfected into the Eca109 and EC9706 cell lines. Luciferase activity was determined $48 \mathrm{~h}$ following transfection using a Luciferase Reporter Assay System (Promega, Corp.). The results were from three independent repeated experiments.

Dual-luciferase reporter assay. A dual-luciferase reporter assay system was performed to investigate the interaction between miR-18a-5p and PART1 or SOX6 in the Eca109 and EC9706 cell lines. Recombinant vectors, pmirGLO-PART1-WT and pmirGLO-PART1-MUT, as well as pmirGLO-SOX6-WT and pmirGLO-SOX6-MUT (TsingKe Biological Technology) along with the miR-18a-5p mimic and NC mimic were transfected into the Eca109 and EC9706 cell lines using Lipofectamine ${ }^{\mathrm{TM}}$ 2000, respectively. Luciferase activity was determined using the Dual-Luciferase Reporter Assay System (Promega Corp.), $48 \mathrm{~h}$ following transfection, according to the manufacturer's protocol. The results were from three independent repeated experiments.

RNA immunoprecipitation (RIP). A RIP assay was performed in the Eca109 and EC9706 cell lines using a RNA-binding protein IP kit (EMD Millipore) according to the manufacturer's protocol. Briefly, RIP lysates were prepared from the Eca109 and EC9706 cell lines transfected with miR-18a-5p mimic or $\mathrm{NC}$ mimic, and then subjected to IP using either $5 \mu 1$ normal mouse IgG or $5 \mu 1$ anti-ago2 antibody and the Mana RIP ${ }^{\mathrm{TM}}$ RNA-binding Protein IP kit. PART1 and miR-18a-5p enriched on the beads was determined using RT-qPCR and corresponding specific primers. The results were from three independent repeated experiments.

Western blot analysis. Total proteins were extracted from the ESCC cell lines using RIPA lysis (Beijing Solarbio Science and Technology Co., Ltd.), while the concentration was measured using the Bradford method. The proteins were separated using $10 \%$ SDS-PAGE, transferred to PVDF membranes (EMD Millipore), and then blocked with skimmed milk and incubated with primary antibodies against SOX6 (cat. no. ab84880), 
$\beta$-catenin (cat. no. ab22656), c-myc (cat. no. ab17355) and $\beta$-actin (cat. no. ab8226) (1:200 dilution, Abcam) overnight at room temperature. Subsequently, the membrane was incubated with the secondary antibody (cat. no. TA130005) (1:5,000 dilution, OriGene Technologies, Inc.). Finally, enhanced chemiluminescence reagents (Beyotime Institutes of Biotechnology) were used to develop the protein signal. The quantification of the blots was analyzed using ImageJ software (v.1.8.0) (National Institutes of Health, USA). The data were from three independent repeated experiments.

Statistical analysis. GraphPad Prism v8.0 software (GraphPad Software, Inc.) was used to analyze all the experimental data. Data are presented as the mean with standard deviation. The association between PART1, SOX6 and miR-18a-5p and the clinicopathological features of the ESCC cases were determined using a $\chi^{2}$ test. High and low expression was based on the median value. When the expression level was more than the median value, the level was defined as high expression; when the expression level was less than the median value, low expression was indicated. Spearman was used to investigate the non-parametric data, and Pearson was performed to analyze the parametric data. Survival analysis was performed using the log-rank test, and survival curves were drawn using Kaplan-Meier. For the matched samples, the data were analyzed using Wilcoxon signed rank, and for non-matched samples, the data were compared by Mann-Whitney test. Comparison between two groups was determined using a Student's t-test, and the comparison of $>3$ groups was determined using one-way ANOVA, and then Bonferroni test was selected for further statistical assay when data sets contained $>3$ groups. $\mathrm{P}<0.05$ was considered to indicate a statistically significant difference.

\section{Results}

Low expression level of PART1 and SOX6 in ESCC tissues and cells is associated with prognosis in the patients with ESCC. To investigate the level of PART1 and SOX6 in ESCC tissues and cells, as well as their clinical value, The Cancer Genome Atlas (TCGA) database, and the GEO dataset were analyzed. The data from TCGA database and GEO dataset revealed that the level of PART1 in ESCA tissues was significantly lower compared with that in normal tissues $(\mathrm{P}<0.05)$ (Fig. 1A and B), which was further verified using RT-qPCR in 75 ESCC samples and matched adjacent normal samples $(\mathrm{P}<0.0001)$ (Fig. 1C). In addition, the GEO GSE111011 dataset revealed that ESCC tissues exhibited lower SOX6 mRNA expression levels compared with that in paired adjacent normal tissues $(\mathrm{P}<0.001)$ (Fig. 1D), and similar results were found using RT-qPCR in 75 ESCC samples and matched adjacent normal samples $(\mathrm{P}<0.0001)$ (Fig. 1E). Furthermore, RT-qPCR demonstrated that the ESCC cell lines (Eca109, EC9706, TE1, KYSE70 and KYSE450) had significantly lower levels of PART1 and SOX6 compared with those in the Het-1A normal esophageal epithelial cell line $(\mathrm{P}<0.01)$ (Fig. $1 \mathrm{~F}$ and $\mathrm{G})$. Correlation analysis showed that PART1 expression level was positively correlated with SOX6 expression level in ESCA tissues (Fig. 1H and I). To further determine the association between PART1 and SOX6 levels with prognosis in patients with ESCC, a log-rank test was used. The results revealed that patients with low levels of PART1 and SOX6 exhibited shorter survival times compared with those with high expression levels of PART1 and SOX6 (Fig. 1J-L). The data suggest that PART1 and SOX6 may participate in the development and progression of ESCC and may be novel prognostic factors in patients with ESCC.

Associations between PART1 and SOX6 levels and the clinicopathological features and prognosis in ESCC. To further investigate the roles of PART1 and SOX6 in ESCC development and progression, GraphPad Prism v8.0 software was used to investigate the association between PART1 and SOX6 level and the clinicopathological features in the patients with ESCC. It was found that low expression levels of PART1 and SOX6 were both associated with higher TNM stage and positive lymph node metastasis (all $\mathrm{P}<0.05$ ); however, there was no association with sex, age, tumor diameter and differentiation degree (all P>0.05) (Tables II and III). The data suggest that PART1 and SOX6 may be novel predictive factors for TNM stage and lymph node metastasis in ESCC.

lncRNA PART1 is a direct transcriptional target of FOXP2 in the ESCC cell lines. To further investigate which factor affects PART1 expression in ESCC, direct targets of PART1 were determined. The upstream promoter sequence $(\sim 2 \mathrm{~kb}$ upstream) of PART1 was detected using promoter analysis tools (UCSC and JASPAR) and the binding site of FOXP2 (ATGTAAACAAT) was found to be within the upstream promoter region of PART1 (Fig. 2A). To preliminarily investigate the expression level of FOXP2 in ESCC, RT-qPCR was used and the results revealed that the FOXP2 mRNA expression level in 75 ESCC tissues was significantly lower compared with that in the matched adjacent normal tissues (Fig. 2B). Correlation analysis demonstrated that PART1 level was positively correlated with FOXP2 mRNA expression level in the ESCA tissues (Fig. 2C). Further investigation revealed that the expression level of FOXP2 was significantly reduced by FOXP2 siRNAs (si-FOXP2\#1 and \#2), and si-FOXP2\#2 was more effective at reducing FOXP2 expression level in the ESCC cell lines (Fig. 2D), whereas pcDNA3.1-FOXP2 significantly increased the expression level of FOXP2 in the ESCC cell lines (Fig. 2E). Based on these results, si-FOXP2\#2 significantly suppressed the expression level of PART1 in the ESCC cell lines; however, FOXP2 upregulation significantly increased the expression level of PART1 (Fig. 2F and G). Then, it was investigated whether FOXP2 directly binds to the predicted promoter region in PART1. The fragments containing WT or MUT binding sites were constructed using the pGL3-basic vector. As expected, the luciferase activity was notably enhanced following co-transfection with pcDNA3.1-FOXP2 and pGL3-PART1-promoter-WT in the Eca109 and EC9706 cell lines; however, there was no effect following co-transfection with pcDNA3.1-FOXP2 and pGL3-PART1-promoter-MUT (Fig. 2H and I). These data suggest that the PART1 expression level may be affected by FOXP2 in ESCC cells.

lncRNA PART1 suppresses cell proliferation and invasion in the ESCC cell lines. To investigate the role of PART1 in the 

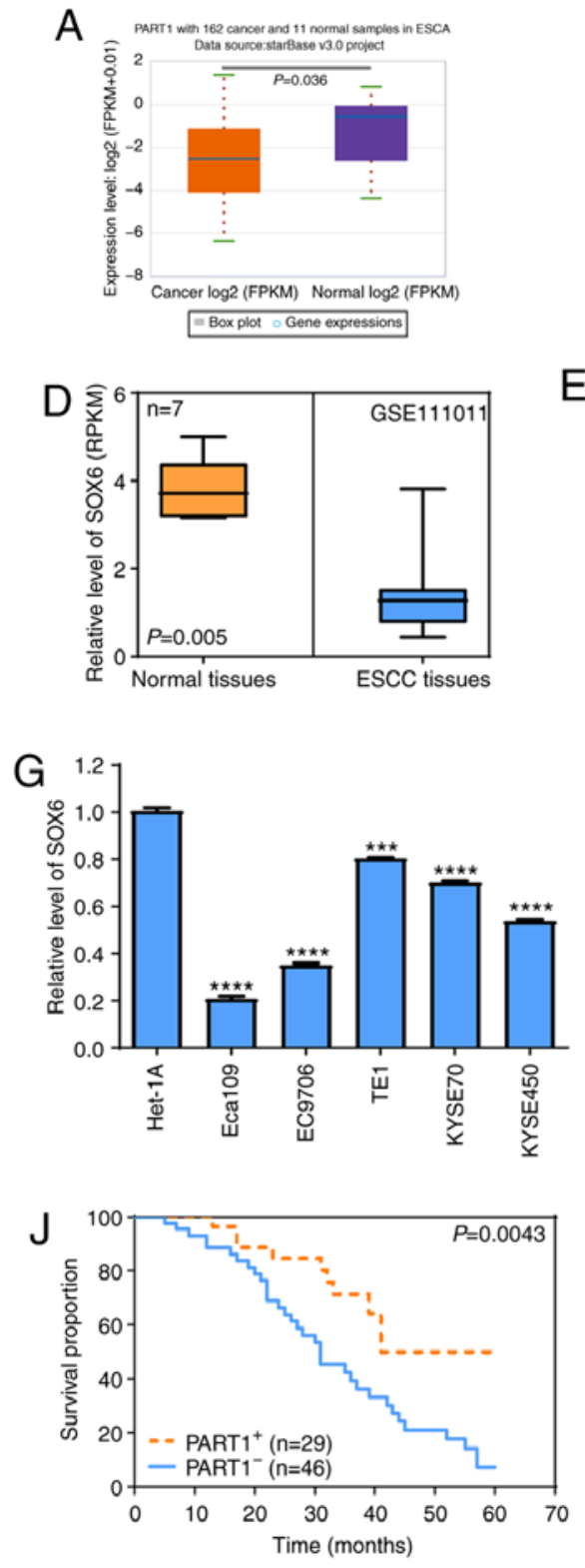
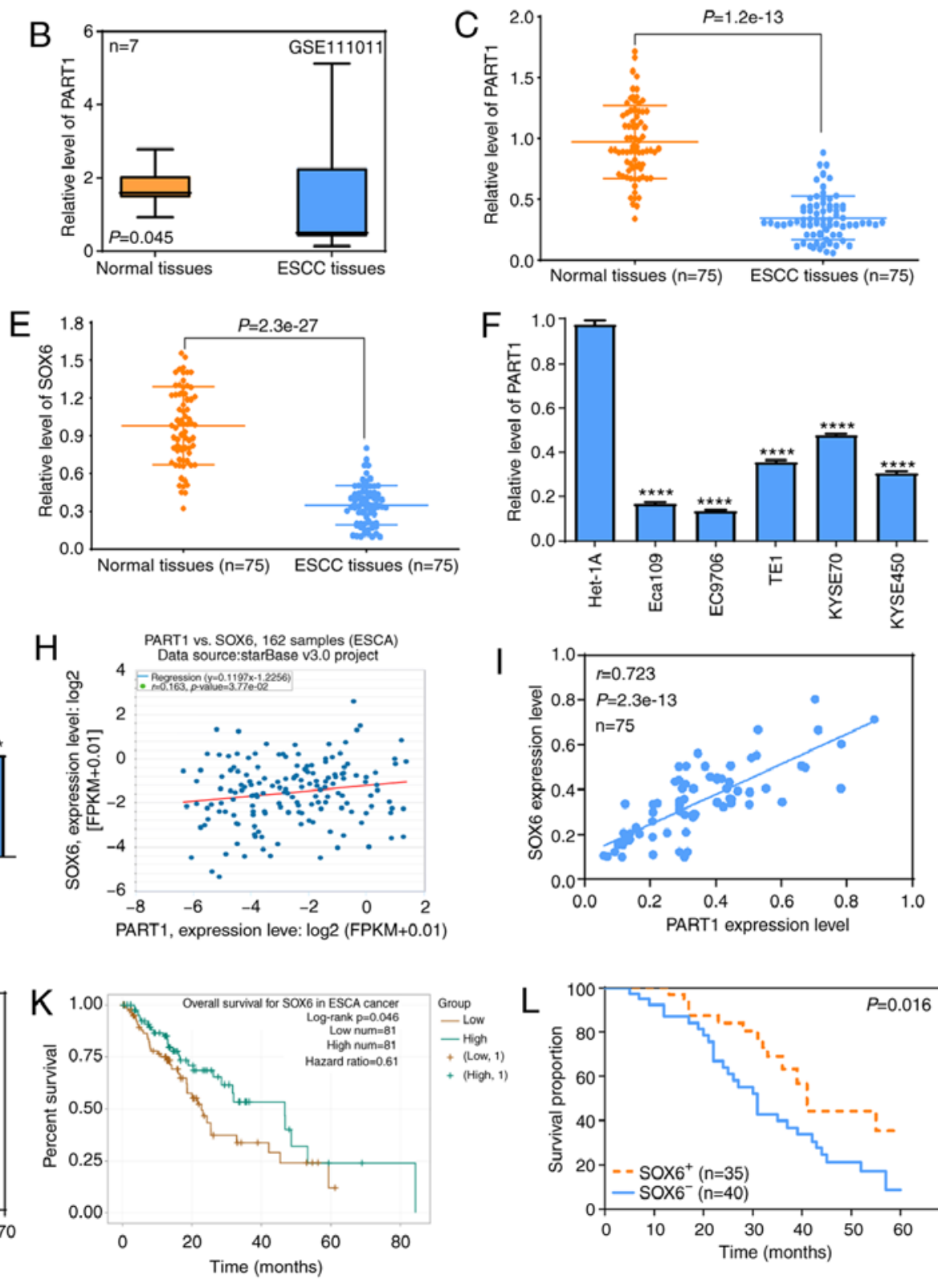

Figure 1. Expression levels of PART1 and SOX6 in ESCC tissues and cells are associated with prognosis in patients with ESCC. (A) StarBase online software was used to determine the expression level of PART1 in 162 ESCA and 11 normal tissues. (B) GEO GSE111011 dataset was analyzed to determine the expression level of PART1 in 7 ESCC and matched normal tissues. P $<0.05$, as investigated by paired t-test. (C) RT-qPCR assay was used to determine the expression level of PART1 in 75 ESCC and paired normal tissues. The data were investigated by Wilcoxon matched-pairs signed-rank sum test. (D) The GEO GSE111011 dataset was used to detect the expression level of SOX6 in 7 ESCC and matched normal tissues, which was investigated by paired t-test. (E) RT-qPCR assay was used to investigate the expression level of SOX6 in 75 ESCC and paired normal tissues, which was examined by paired t-test. (F and G) Expression levels of PART1 and SOX6 in the ESCC cell lines (Eca109, EC9706, TE1, KYSE70 and KYSE450) and the Het-1A normal esophageal epithelial cell line. ${ }^{* * * *} \mathrm{P}<0.0001$ vs. Het-1A, which was examined by one-way ANOVA and Bonferroni test. (H) The StarBase online software was used to investigate the correlation between PART1 and SOX6 mRNA expression levels in ESCA tissues. (I) Spearman's correlation results between PART1 and SOX6 mRNA expression levels in 75 ESCC and matched adjacent normal samples. (J) Log-rank test was used to determine the association between PART1 expression level and the survival times in patients with ESCC. (K) The StarBase online software was used to investigate the association between SOX6 expression level and prognosis in patients with ESCC. (L) Log-rank test was used to investigate the association between SOX6 expression level and the survival times in patients with ESCC. RT-qPCR, reverse transcription-quantitative PCR; ESCA, esophageal cancer; ESCC, esophageal squamous cell carcinoma; PART1, prostate androgen regulated transcript 1; SOX6, SRY-box transcription factor 6.

proliferation and invasion of the ESCC cell lines, CCK-8 and Matrigel assays were used. It was found that pcDNA3.1-PART1 significantly upregulated PART1 expression level in the Eca109 and EC9706 cell lines $(\mathrm{P}<0.0001)$ (Fig. 3A). In addition, PART1 upregulation markedly suppressed the cell proliferation of the Eca109 and EC9706 cell lines compared with that in the pcDNA3.1 group $(\mathrm{P}<0.05)$ (Fig. 3B). The Matrigel assay, using a Transwell chamber, revealed that PART1 overexpression significantly inhibited cell invasion in the Eca109 and EC9706 cell lines (Fig. 3C and D). Conversely, three siRNAs against PART1 were used to downregulate the expression level of PART1 in the Eca109 and EC9706 cell lines, as expected, all three PART1 siRNAs significantly reduced the expression level of PART1, in which PART1 siRNA\#1 was the most 
Table II. Associations of PART1 expression with clinicopathological features in the ESCC cases $(\mathrm{N}=75)$.

\begin{tabular}{|c|c|c|c|c|c|}
\hline \multirow[b]{2}{*}{ Features } & \multirow[b]{2}{*}{$\mathrm{n}$} & \multicolumn{2}{|c|}{$\begin{array}{c}\text { PART1 } \\
\text { expression }\end{array}$} & \multirow[b]{2}{*}{$\chi^{2}$} & \multirow[b]{2}{*}{ P-value } \\
\hline & & High & Low & & \\
\hline \multicolumn{6}{|l|}{ Sex } \\
\hline Male & 49 & 18 & 31 & 0.222 & 0.637 \\
\hline Female & 26 & 11 & 15 & & \\
\hline \multicolumn{6}{|l|}{ Age (years) } \\
\hline$<60$ & 35 & 16 & 19 & & \\
\hline$\geq 60$ & 40 & 13 & 27 & 1.374 & 0.241 \\
\hline \multicolumn{6}{|c|}{ Tumor diameter $(\mathrm{cm})$} \\
\hline$<4$ & 50 & 21 & 29 & & \\
\hline$\geq 4$ & 25 & 8 & 17 & 0.703 & 0.402 \\
\hline \multicolumn{6}{|c|}{ Differentiation degree } \\
\hline High/moderate & 44 & 20 & 24 & & \\
\hline Poor & 31 & 9 & 22 & 2.068 & 0.150 \\
\hline \multicolumn{6}{|l|}{ TNM staging } \\
\hline I-II & 40 & 21 & 19 & & \\
\hline III-IV & 35 & 8 & 27 & 6.916 & 0.009 \\
\hline \multicolumn{6}{|c|}{ Lymph node metastasis } \\
\hline Yes & 23 & 4 & 19 & & \\
\hline No & 52 & 25 & 27 & 6.331 & 0.012 \\
\hline
\end{tabular}

PART1, prostate androgen regulated transcript 1; ESCC, esophageal squamous cell carcinoma.

effective (Fig. 3E). A further CCK-8 assay demonstrated that PART1 downregulation significantly promoted cell proliferation in the Eca109 and EC9706 cell lines $(\mathrm{P}<0.05)$ (Fig. 3F). Meanwhile, PART1 knockdown accelerated cell invasion in the Eca109 and EC9706 cell lines (Fig. 3G and H). These data suggest that PART1 plays a pivotal role in the regulation of cell proliferation and invasion of the ESCC cell lines.

miR-18a-5p is the direct target of PART1 in the ESCC cell lines. To investigate the underlying molecular mechanism of PART1 in ESCC progression, IncATLAS online software was used to predict the subcellular localization of PART1. The data revealed that PART1 was primarily localized in the cytoplasm in most of the indicated cell types (Fig. 4A). To validate the predicted result, RT-qPCR was used in the ESCC cell lines. The results demonstrated that PART1 was principally localized in the cytoplasm of the Eca109 and EC9706 cell lines (Fig. 4B). According to the localization of PART1, a proposed ceRNA function of PART1 was suggested. The possible binding sites of miRNA in PART1 were predicted using the LncBase Predicted v.2 software, integrated in DIANA Tools, in which miR-18a-5p was found to contain a target site and was investigated further in the present study (Fig. 4C). To preliminarily elucidate whether PART1 could bind to miR-18a-5p in the ESCC cell lines, an Ago2-RIP experiment was used to verify the direct interaction between PART1 and
Table III. Associations of SOX6 expression with clinicopathological features in the ESCC cases $(\mathrm{N}=75)$.

\begin{tabular}{|c|c|c|c|c|c|}
\hline \multirow[b]{2}{*}{ Features } & \multirow[b]{2}{*}{$\mathrm{n}$} & \multicolumn{2}{|c|}{$\begin{array}{c}\text { SOX6 } \\
\text { expression }\end{array}$} & \multirow[b]{2}{*}{$\chi^{2}$} & \multirow[b]{2}{*}{ P-value } \\
\hline & & High & Low & & \\
\hline \multicolumn{6}{|l|}{ Sex } \\
\hline Male & 49 & 20 & 29 & 1.944 & 0.163 \\
\hline Female & 26 & 15 & 11 & & \\
\hline \multicolumn{6}{|l|}{ Age (years) } \\
\hline$<60$ & 35 & 19 & 16 & & \\
\hline$\geq 60$ & 40 & 16 & 24 & 1.531 & 0.216 \\
\hline \multicolumn{6}{|c|}{ Tumor diameter (cm) } \\
\hline$<4$ & 50 & 26 & 24 & & \\
\hline$\geq 4$ & 25 & 9 & 16 & 1.714 & 0.190 \\
\hline \multicolumn{6}{|c|}{ Differentiation degree } \\
\hline High/moderate & 44 & 24 & 20 & & \\
\hline Poor & 31 & 11 & 20 & 2.655 & 0.103 \\
\hline \multicolumn{6}{|l|}{ TNM staging } \\
\hline I-II & 40 & 25 & 15 & & \\
\hline III-IV & 35 & 10 & 25 & 8.634 & 0.003 \\
\hline \multicolumn{6}{|c|}{ Lymph node metastasis } \\
\hline Yes & 23 & 6 & 17 & & \\
\hline No & 52 & 29 & 23 & 5.645 & 0.018 \\
\hline
\end{tabular}

SOX6, SRY-box transcription factor 6; ESCC, esophageal squamous cell carcinoma.

miR-18a-5p. The results showed that PART1 and miR-18a-5p expression levels in the anti-Ago 2 antibody group were significantly higher compared with that in the IgG group $(\mathrm{P}<0.0001)$ (Fig. 4D and E). Notably, PART1 enrichment in the miR-18a-5p mimic group was significantly higher compared with that in the NC mimic group $(\mathrm{P}<0.0001)$ (Fig. 4F). These findings suggested that PART1 and miR-18a-5p exhibited a direct interaction in the ESCC cell lines via the Ago2-dependent pathway. In additon, a dual-luciferase reporter assay revealed that the relative luciferase intensity was significantly reduced following co-transfection with PART1-WT and miR-18a-5p mimic, but not in the PART1-MUTgroup, in the Eca109 and EC9706 cell lines (Fig. 4G). Simultaneously, PART1 overexpression significantly reduced the expression level of miR-18a-5p (Fig. 4H), whereas PART1 silencing significantly promoted miR-18a-5p expression in the Eca109 and EC9706 cell lines (Fig. 4I). These findings indicated that PART1 directly regulated the expression level of miR-18a-5p in the ESCC cell lines.

SOX6 is the direct target of miR-18a-5p in the ESCC cell lines. It is well-known that miRNA functions as either an oncogene or tumor suppressor, depending on its downstream target gene. To further investigate the role of miR-18a-5p in ESCC progression, its expression level and downstream target genes were determined. The StarBase online software was used to investigate 
A
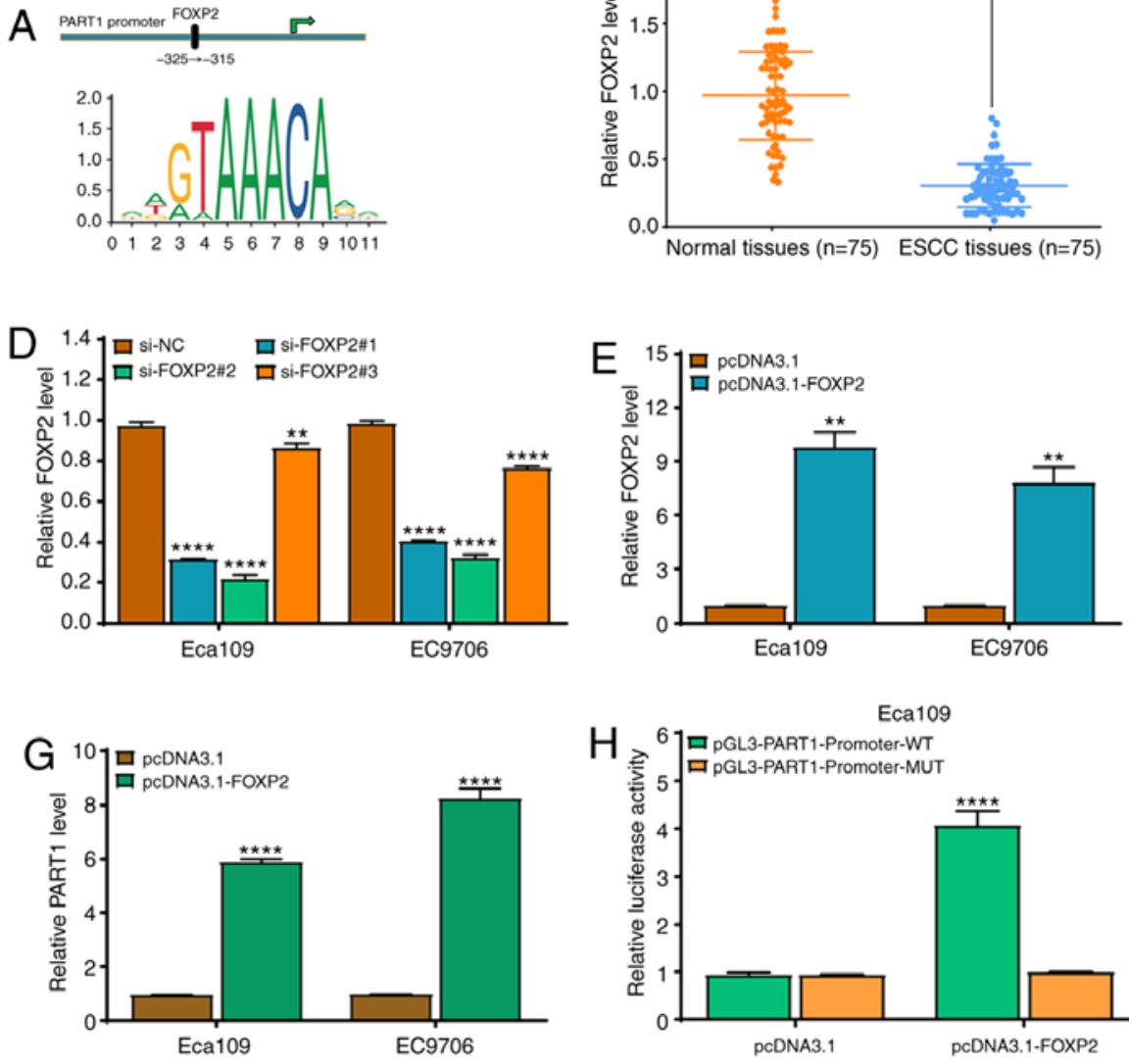
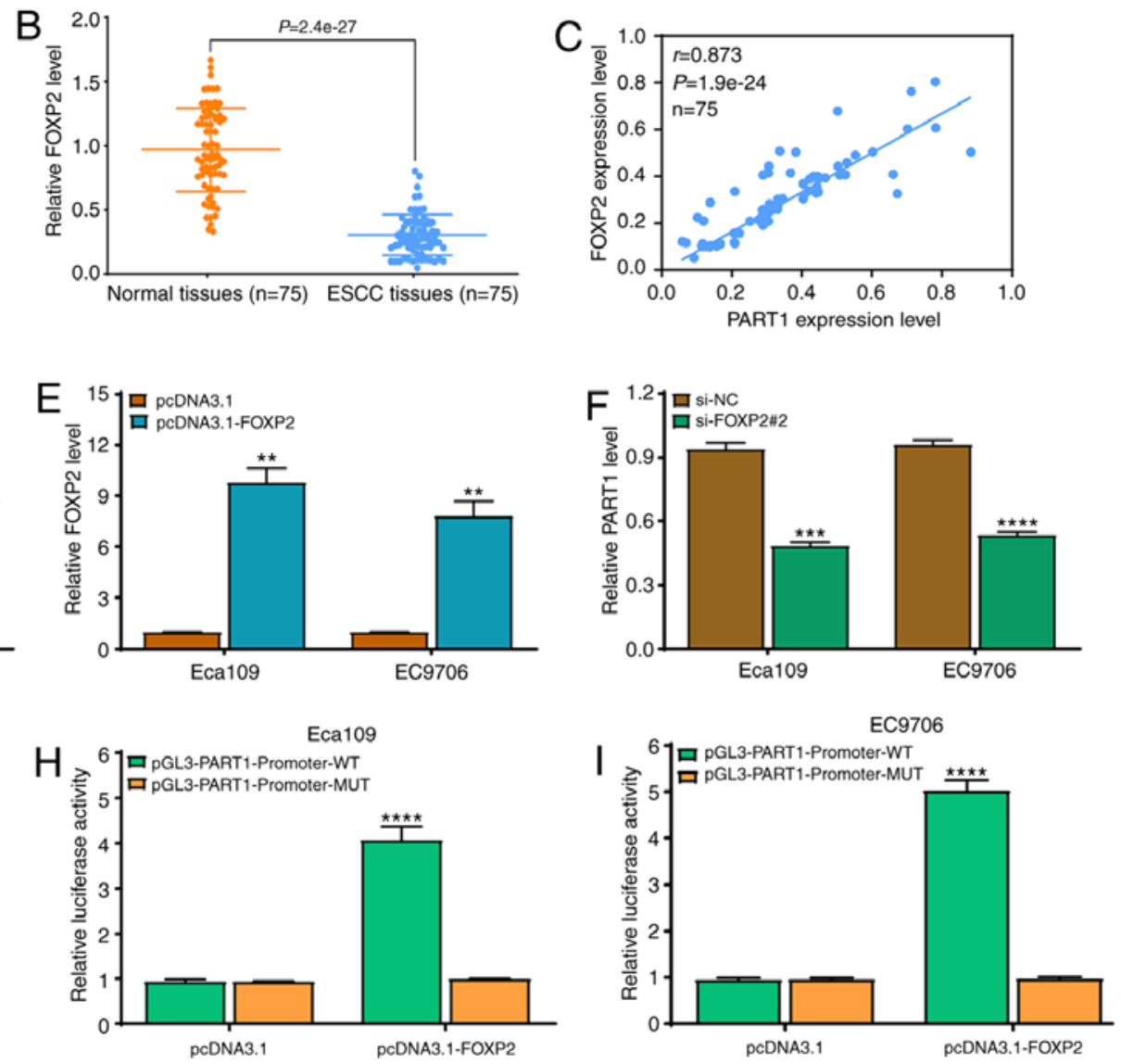

Figure 2. Transcriptional factor, FOXP2, regulates PART1 expression level in the ESCC cell lines. (A) A predicted FOXP2 binding site in the PART1 promoter region. (B) RT-qPCR assay was used to analyze the expression level of FOXP2 in 75 ESCC and matched normal tissues. P $<0.0001$, as investigated by paired t-test. (C) Spearman's correlation result between PART1 and FOXP2 mRNA expression levels in 75 ESCC and matched normal samples. (D) A total of 3 siRNAs targeting FOXP2 were used to downregulate FOXP2 expression level in the ESCC cell lines and validated using RT-qPCR; si-FOXP2\#2 was the most effective. ${ }^{* *} \mathrm{P}<0.01$ and ${ }^{* * * * *} \mathrm{P}<0.0001$ vs. the si-NC group. (E) pcDNA3.1-FOXP2 significantly promoted the expression level of FOXP2 in the ESCC cell lines as determined by RT-qPCR. ${ }^{* *} \mathrm{P}<0.01$ vs. the pcDNA3.1 group; the data were examined by one-way ANOVA and Bonferroni test. (F) si-FOXP2\#2 significantly downregulated the expression level of PART1 in the ESCC Eca109 and EC9706 cell lines as determined by RT-qPCR. ${ }^{* * *} \mathrm{P}<0.001$ and ${ }^{* * * * *} \mathrm{P}<0.0001$ vs. the si-NC group. (G) pcDNA3.1-FOXP2 significantly promoted the expression level of PART1 in the ESCC cell lines as determined by RT-qPCR ${ }_{* * * * *} \mathrm{P}<0.0001$ vs. the pcDNA3.1 group. (H and I) Luciferase reporter assay was used to determine the luciferase activity in pGL3-PART1-promoter-WT or pGL3-PART1-promoter-MUT in the FOXP2-overexpressing (H) Eca109 and (I) EC9706 cell lines. ${ }^{* * * *} \mathrm{P}<0.0001$ vs. MUT. RT-qPCR, reverse transcription-quantitative PCR; WT, wild-type; MUT, mutant; si, small interfering; NC, negative control; ESCC, esophageal squamous cell carcinoma; PART1, prostate androgen regulated transcript 1. (E-I) All data were examined by independent-sample t-test.

the expression level of miR-18a-5p in 162 ESCA tissues and 11 normal samples, and it was found that miR-18a-5p expression level was markedly higher compared with that in normal samples (Fig. 5A), which was further verified using the GEO GSE43732 dataset in 119 ESCC samples and matched adjacent normal samples (Fig. 5B). To validate the data from bioinformatics analysis, RT-qPCR was used to detect the miR-18a-5p expression level in 75 ESCC samples and paired adjacent normal samples. It was found that 75 ESCC samples displayed higher expression level of miR-18a-5p compared with that in the paired adjacent normal samples $(\mathrm{P}<0.0001)$ (Fig. 5C), and similar data were obtained from a panel of ESCC cell lines and the Het-1A normal esophageal epithelial cell line (Fig. 5D). Notably, the miR-18a-5p expression level was associated with TNM stage and lymph node metastasis in patients with ESCC (Table IV). These findings suggested that miR-18a-5p may participate in ESCC development and progression. To further investigate its downstream target genes, Targetscan and miRDB online software programs were used to predict the possible binding sites of miR-18a-5p in the SOX6 3'-untranslated region (UTR) (Fig. 5E). A dual-luciferase reporter assay revealed that the relative luciferase intensity was significantly reduced by co-transfecting with SOX6-WT and miR-18a-5p mimic, but not in the SOX6-MUT group, in the Eca109 and EC9706 cell lines (Fig. 5F). Correlation analysis revealed that the miR-18a-5p expression level was negatively correlated with the SOX6 expression level in the 75 ESCC samples (Fig. 5G). In addition, miR-18a-5p inhibitor significantly suppressed miR-18a-5p expression in the Eca109 and EC9706 cell lines (Fig. 5H), whereas miR-18a-5p mimic significantly promoted miR-18a-5p expression level in the Eca109 and EC9706 cell lines (Fig. 5I). Simultaneously, the miR-18a-5p inhibitor significantly increased the mRNA expression level of SOX6 in the Eca109 and EC9706 cell lines (Fig. 5J), whereas miR-18a-5p mimic significantly suppressed the mRNA expression level of SOX6 in the Eca109 and EC9706 cell lines (Fig. 5K). These findings indicated that miR-18a-5p directly affected the expression level of SOX6 in the ESCC cell lines. 

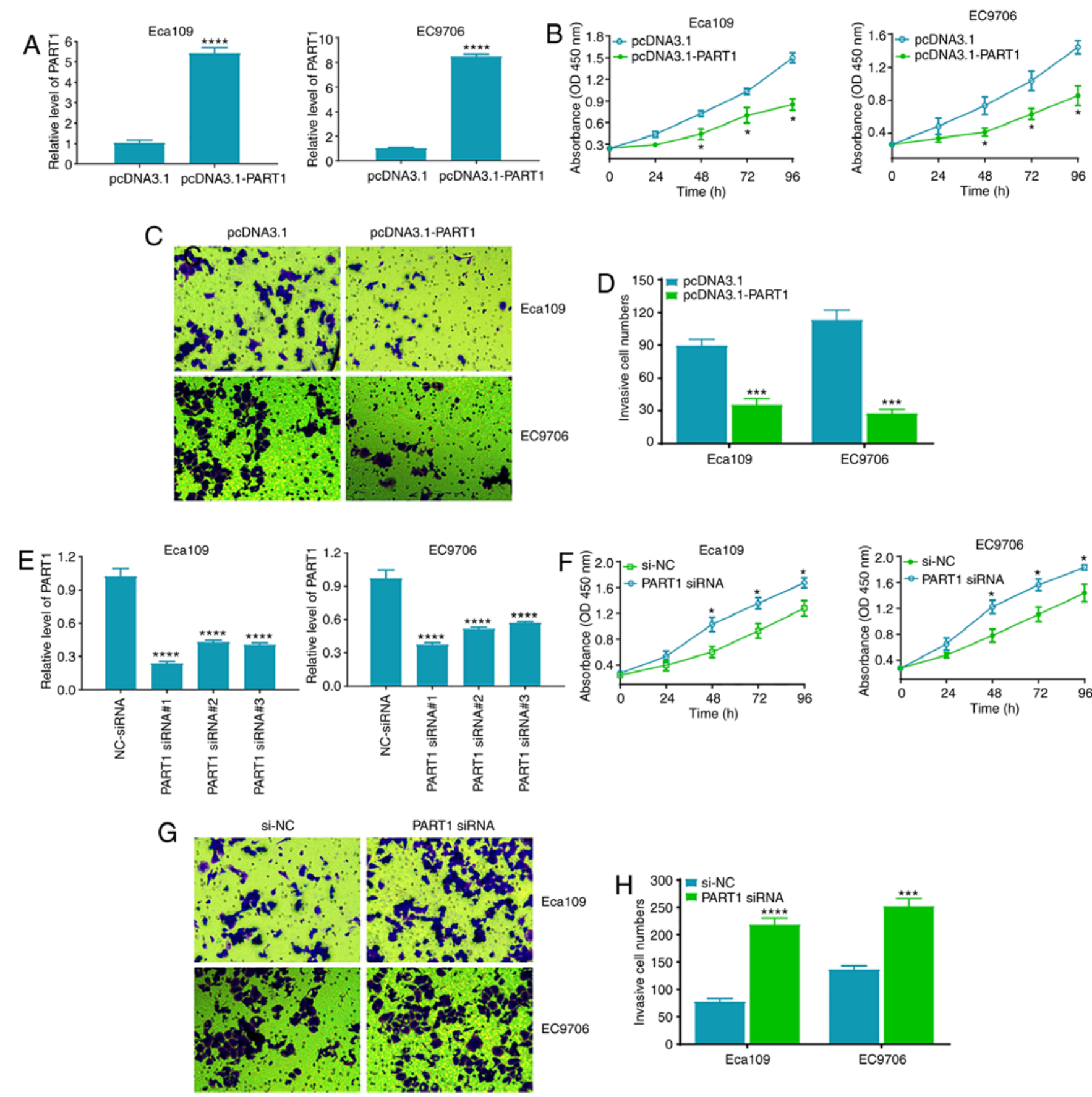

Figure 3. Exogenous expression of PART1 suppresses ESCC cell proliferation and invasion. (A) RT-qPCR assay was used to analyze PART1 expression level in the Eca109 and EC9706 cell lines, $48 \mathrm{~h}$ following transfection with pcDNA3.1 and pcDNA3.1-PART1. (B) CCK-8 assay was used to analyze cell proliferation in the Eca109 and EC9706 cell lines, at 24, 48, 72 and $96 \mathrm{~h}$ following transfection with pcDNA3.1 and pcDNA3.1-PART1. (C) A Matrigel assay was used to analyze invasion ability in the Eca109 and EC9706 cell lines, $48 \mathrm{~h}$ following transfection with pcDNA3.1 and pcDNA3.1-PART1. (D) Statistical analysis of the number of invasive cells in the Eca109 and EC9706 cell lines transfected with pcDNA3.1 and pcDNA3.1-PART1. ${ }^{*} \mathrm{P}<0.05,{ }^{* * * *} \mathrm{P}<0.001$ and ${ }^{* * * * *} \mathrm{P}<0.0001$ vs. the pcDNA3.1 group. (E) RT-qPCR assay was used to analyze PART1 expression level in the Eca109 and EC9706 cell lines, $48 \mathrm{~h}$ following transfection with si-NC and PART1 siRNA\#1, 2 and 3, and the data were examined by one-way ANOVA and Bonferroni test. (F) CCK-8 assay was used to analyze cell proliferation in the Eca109 and EC9706 cell lines, at 24, 48, 72 and $96 \mathrm{~h}$ following transfection with si-NC and PART1 siRNA. (G) Matrigel assay was used to analyze invasion in the Eca109 and EC9706 cell lines, $48 \mathrm{~h}$ following transfection with si-NC and PART1 siRNA. (H) Statistical analysis of the number of invasive cells in the Eca109 and EC9706 cell lines transfected with si-NC and PART1 siRNA. ${ }^{*} \mathrm{P}<0.05,{ }^{* * * *} \mathrm{P}<0.001$ and ${ }^{* * * * *} \mathrm{P}<0.0001$ vs. the si-NC group. CCK-8, Cell Counting Kit-8; RT-qPCR, reverse transcription-quantitative PCR; si, small interfering; NC, negative control; ESCC, esophageal squamous cell carcinoma; PART1, prostate androgen regulated transcript 1. (A-D and F-H) All data were examined by independent-sample t-test.

Role of the PART1/miR-18a-5p/SOX6 signaling axis in cell proliferation and invasion of the ESCC cell lines. To investigate the potential role of the PART1/miR-18a-5p/SOX6 signaling axis in cell proliferation and invasion of the ESCC cell lines, miR-18a-5p inhibitor or mimic alone, combined with PART1 siRNA and SOX6 siRNA or pcDNA3.1-PART1 and pcDNA3.1-SOX6 were used to transfect the Eca109 and EC9706 cell lines, and then CCK-8 and Matrigel assays were performed. It was found that miR-18a-5p inhibitor significantly blocked cell proliferation and invasion in the Eca109 
A

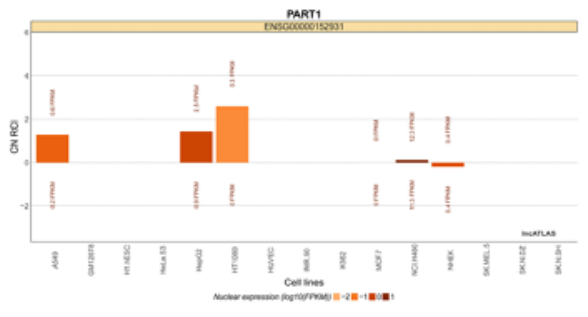

B

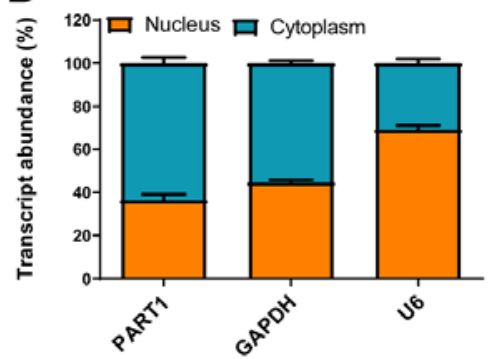

EC9706

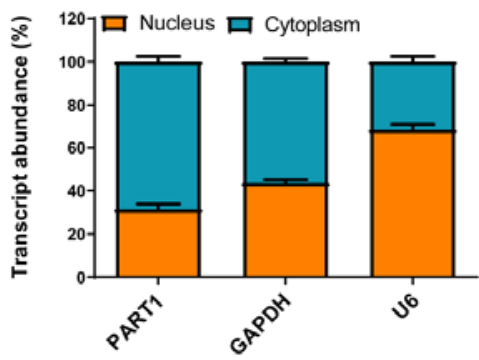

C

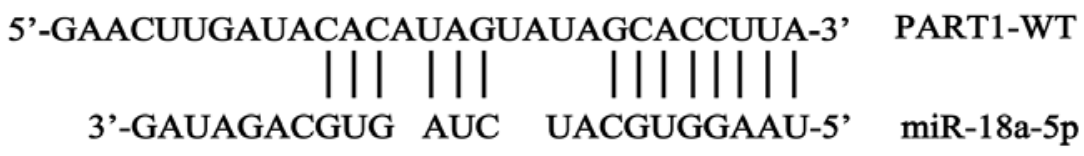

\section{5'-GAACUUGAUAGUGAAUCUAUACGUGGAAU-3' PART1-Mut}

$\mathrm{D}$

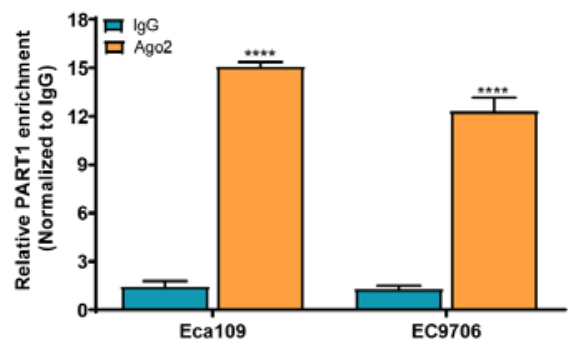

$\mathrm{E}$

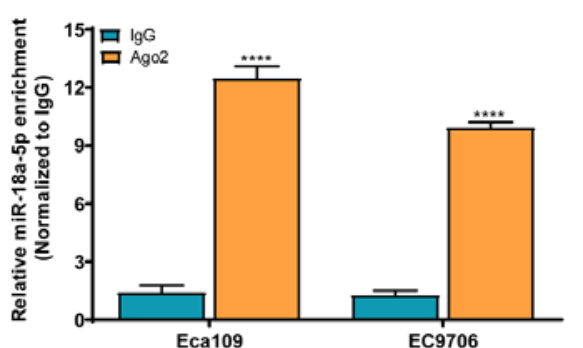

$\mathrm{F}$

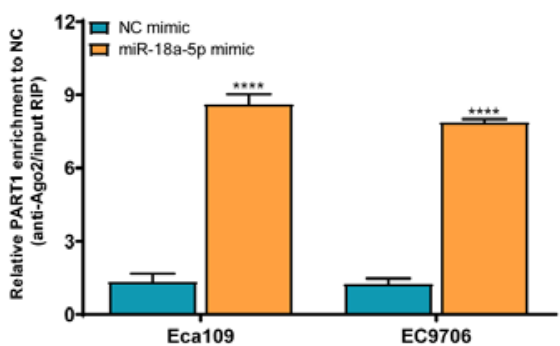

G

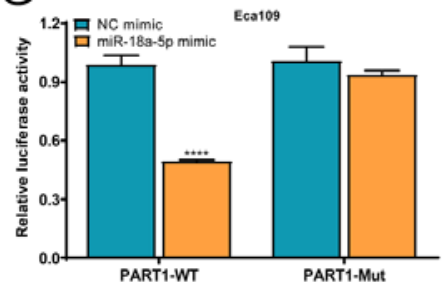

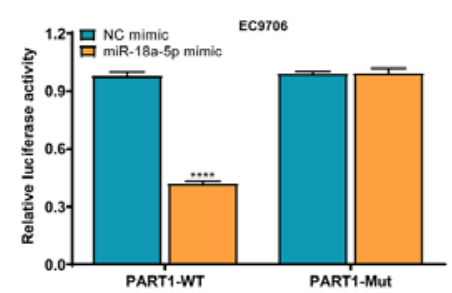

$\mathrm{H}$

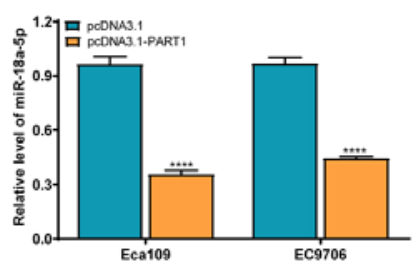

I

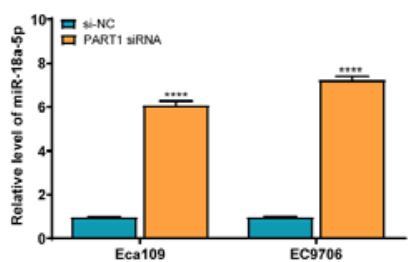

Figure 4. PART1 affects the expression level of miR-18a-5p in the ESCC cell lines. (A) The localization of PART1 was predicted using the lncATLAS online software. (B) RT-qPCR assay was performed to investigate the expression level of PART1 in the nucleus and cytoplasm of the Eca109 and EC9706 cell lines. U6 and GAPDH were used as the endogenous controls. (C) The predicted miR-18a-5p binding sites in the PART1 transcript. Relative enrichment of (D) PART1 and (E) miR-18a-5p using RIP and anti-Ago2 antibody in the Eca109 and EC9706 cell lines. The fold enrichment was normalized to IgG, as the negative control. ${ }^{* * * *} \mathrm{P}<0.0001$ vs. IgG group. (F) Relative enrichment of PART1 in the Eca109 and EC9706 cell lines transfected with NC or miR-18a-5p mimic. ${ }^{* * * *} \mathrm{P}<0.0001$ vs. NC mimic. (G) The luciferase activity assay in the Eca109 and the EC9706 cell lines co-transfected with pmirGLO-PART1-WT or pmirGLO-PART1-MUT together with NC or miR-18a-5p mimic. ${ }^{* * * *} \mathrm{P}<0.0001$ vs. NC mimic. (H) qPCR assay was used to analyze the expression level of miR-18a-5p in the Eca109 and EC9706 cell lines transfected with pcDNA3.1 or pcDNA3.1-PART1. ${ }^{* * * *} \mathrm{P}<0.0001$ vs. the pcDNA3.1 group. (I) qPCR assay was used to analyze the expression levels of miR-18a-5p in the Eca109 and EC9706 cell lines transfected with si-NC or PART1 siRNA. ${ }^{* * * *} \mathrm{P}<0.0001$ vs. the si-NC group. Data are presented as the mean \pm SD and were obtained from three independent repeats. WT, wild-type; MUT, mutant; miR, microRNA; NC, negative control; RT-qPCR, reverse transcription-quantitative PCR; PART1, prostate androgen regulated transcript 1. (D-I) All data were examined by independent-sample t-test.

and EC9706 cell lines, which was partly reversed by PART1 and SOX6 siRNA (Fig. 6A-C). Notably, miR-18a-5p inhibitor triggered the upregulation of SOX6 proteins and the downregulation of $\beta$-catenin and c-myc proteins, which was also partly reversed by PART1 and SOX6 siRNA (Fig. 6D). Conversely, miR-18a-5p mimic markedly accelerated cell proliferation and invasion, while pcDNA3.1-PART1 and pcDNA3.1-SOX6 both notably suppressed the promoting effect of miR-18a-5p mimic on cell proliferation and invasion in the Eca109 and EC9706 cell lines (Fig. 6E-G). Notably, miR-18a-5p mimic triggered the downregulation of SOX6 proteins and the upregulation of $\beta$-catenin and c-myc proteins, which was also partly reversed by pcDNA3.1-PART1 and pcDNA3.1-SOX6 (Fig. 6H). These data suggest that the PART1/miR-18a-5p/SOX6/ $\beta$-catenin signaling axis may be a novel therapeutic strategy in patients with ESCC.

\section{Discussion}

An increasing number of studies have demonstrated that lncRNAs play a pivotal role in tumor progression and may be novel diagnostic and therapeutic opportunities for 
A

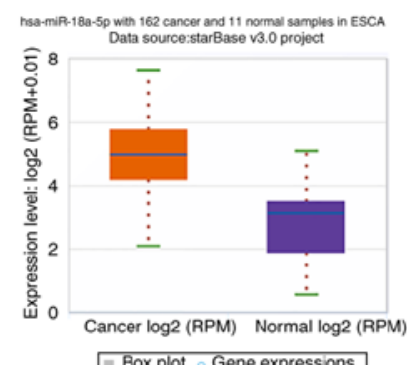

$=$ Box plot $\circ$ Gene expressions

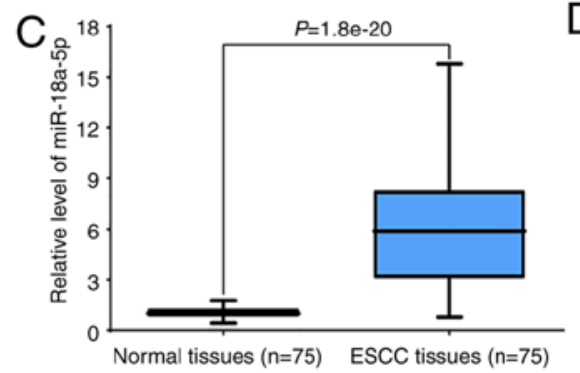

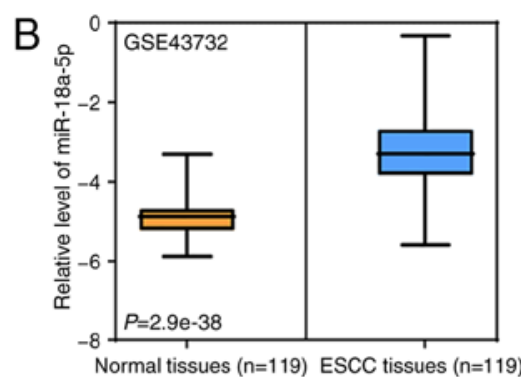

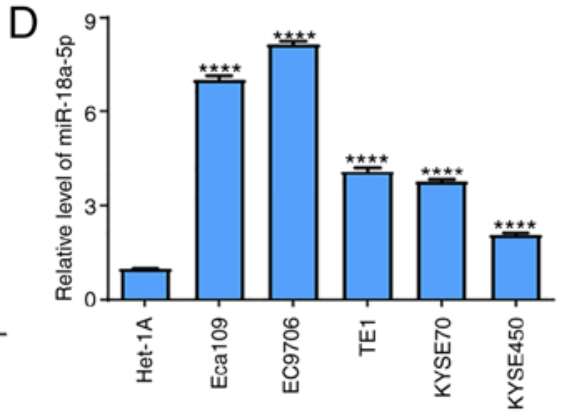

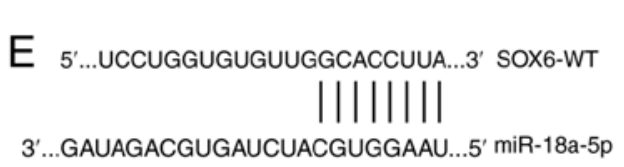

$5^{\prime}$... UCCUGGuguguUgCGUGGAAU...3' SOX6-Mut
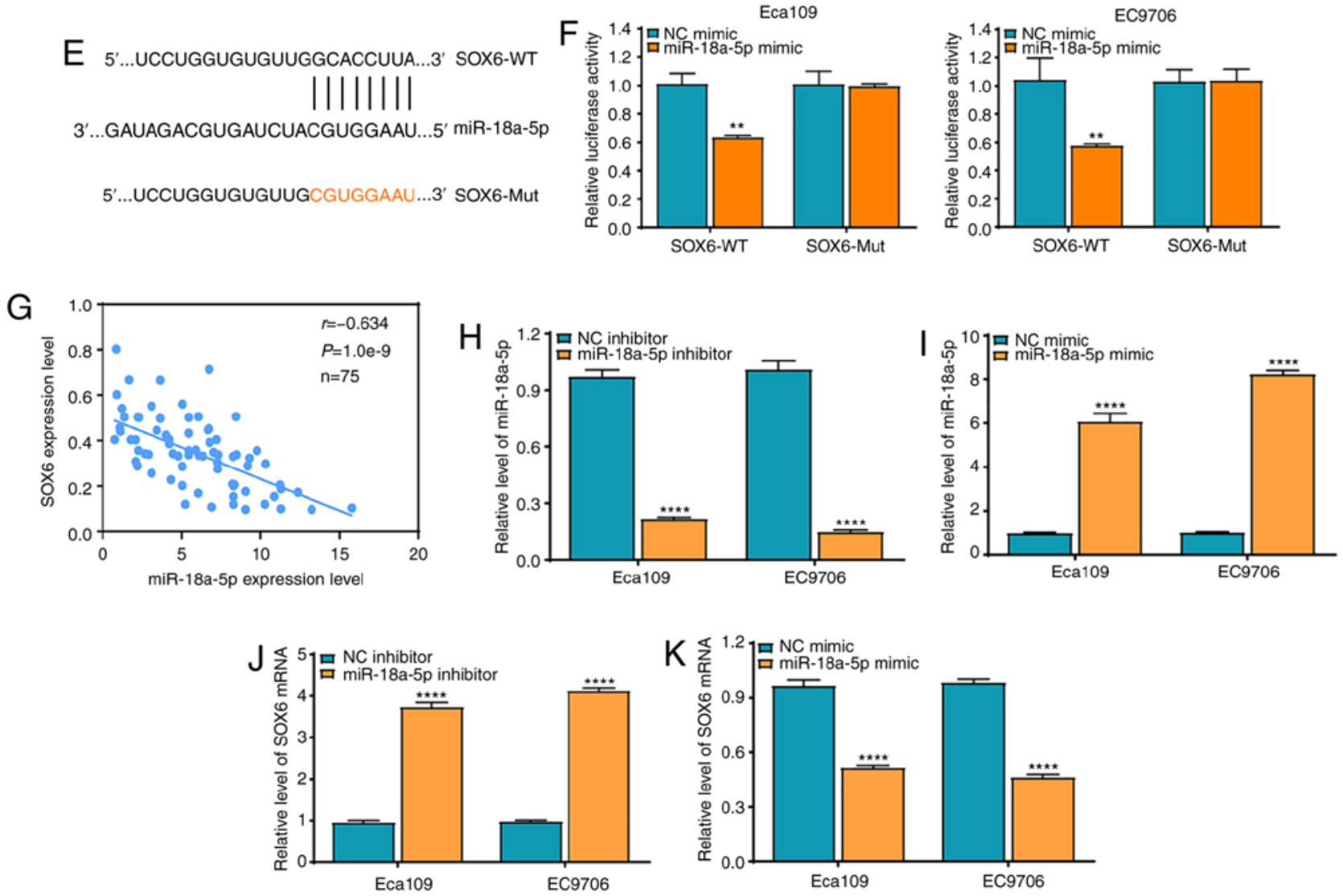

Figure 5. SOX6 is a direct molecular target of miR-18a-5p in the ESCC cell lines. (A) StarBase online software was used to analyze the expression level of miR-18a-5p in 162 ESCA and 11 normal samples. (B) The GEO GSE43732 dataset was used to analyze the expression level of miR-18a-5p in 119 ESCC and matched adjacent normal specimens. (C) RT-qPCR assay was used to analyze the expression level of miR-18a-5p in 75 ESCC and paired adjacent normal tissues. (D) RT-qPCR was used to analyze the expression level of miR-18a-5p in the ESCC cell lines (Eca109, EC9706, TE1, KYSE70 and KYSE450) and the Het-1A normal esophageal epithelial cell line. ${ }^{* * * *} \mathrm{P}<0.0001$ vs. the Het-1A cell line. Data were examined by one-way ANOVA and Bonferroni test. (E) TargetScan and miRDB databases were used to predict the potential binding sites between miR-18a-5p and SOX6. (F) A dual-luciferase reporter assay was performed to investigate the interaction between miR-18a-5p and SOX6. ${ }^{* *} \mathrm{P}<0.01 \mathrm{vs.} \mathrm{NC} \mathrm{mimic.} \mathrm{(G)} \mathrm{Pearson} \mathrm{correlation} \mathrm{test} \mathrm{analysis} \mathrm{between} \mathrm{miR-18a-5p} \mathrm{and}$ SOX6 mRNA expression levels in 75 ESCC and matched adjacent normal samples. (H) miR-18a-5p inhibitor significantly suppressed the miR-18a-5p expression level in the Eca109 and EC9706 cell lines. ${ }^{* * * * *} \mathrm{P}<0.0001$ vs. the NC inhibitor. (I) miR-18a-5p mimic significantly enhanced the miR-18a-5p expression level in the Eca109 and EC9706 cell lines. ${ }^{* * * *} \mathrm{P}<0.0001$ vs. the NC mimic. (J) miR-18a-5p downregulation significantly promoted the expression level of SOX6 in the Eca109 and EC9706 cell lines. ${ }^{* * * * *} \mathrm{P}<0.0001$ vs. the NC inhibitor. (K) miR-18a-5p overexpression significantly suppressed the expression level of SOX6 in the Eca109 and EC9706 cell lines. ${ }^{* * * *} \mathrm{P}<0.0001$ vs. the NC mimic. si, small interfering; NC, negative control; WT, wild-type; MUT, mutant; miR, microRNA; ESCC, esophageal squamous cell carcinoma; SOX6, SRY-box transcription factor 6. (B and C) Data were analyzed using paired t-test; (F and H-K) All data were examined by independent-sample t-test. 
Table IV. Associations of miR-18a-5p expression with clinicopathological features in the ESCC cases $(\mathrm{N}=75)$.

\begin{tabular}{lrrrrr}
\hline & \multicolumn{5}{c}{$\begin{array}{c}\text { miR-18a-5p } \\
\text { expression }\end{array}$} \\
\cline { 3 - 4 } Features & n & High & Low & $\chi^{2}$ & P-value \\
\hline Sex & & & & & \\
$\quad$ Male & 49 & 36 & 13 & 1.943 & 0.163 \\
Female & 26 & 15 & 11 & & \\
Age (years) & & & & & \\
$\quad<60$ & 35 & 20 & 15 & & \\
$\geq 60$ & 40 & 31 & 9 & 3.555 & 0.059 \\
Tumor diameter (cm) & & & & & \\
$<4$ & 50 & 32 & 18 & & \\
$\geq 4$ & 25 & 19 & 6 & 1.103 & 0.294 \\
Differentiation degree & & & & & \\
$\quad$ High/moderate & 44 & 28 & 16 & & \\
Poor & 31 & 23 & 8 & 0.932 & 0.334 \\
TNM staging & & & & & \\
I-II & 40 & 23 & 17 & & \\
III-IV & 35 & 28 & 7 & 4.343 & 0.037 \\
Lymph node metastasis & & & & & \\
$\quad$ Yes & 23 & 20 & 3 & & \\
$\quad$ No & 52 & 31 & 21 & 5.478 & 0.019 \\
\hline
\end{tabular}

ESCC, esophageal squamous cell carcinoma.

numerous patients with cancer. For example, HOTAIR, as a chromatin-regulating IncRNA, was found to function as an oncogene, and its overexpression has been associated with tumor progression, invasion and metastasis $(32,33)$. ANRIL overexpression was associated with rapid tumor progression and shortened survival times in patients with nasopharyngeal carcinoma (34) and hepatocellular carcinoma (35). In addition, prostate androgen regulated transcript 1 (PART1) was found to be significantly upregulated in non-small cell lung cancer (NSCLC), and was associated with shorter overall survival times; therefore, it could serve as an independent prognostic marker in patients with NSCLC with stage I-III (36). Jin et al (24) found that there was a low expression level of PART1 in glioma tissues, which was confirmed using TCGA database and RT-qPCR. PART1, at high expression levels, was associated with advanced stage and predicated poor survival time in patients with prostate cancer (37). These data highlighted the clinical value of lncRNAs in tumors, particularly in diagnosis and for prognosis. Currently, the expression level and the potential clinical value of PART1 in ESCC remain elusive. In the present study, PART1 was found to be expressed at low levels in ESCC tissues and cells, and a low expression level was associated with TNM stage and lymph node metastasis. Notably, patients with ESCC and low PART1 expression level had poorer survival times compared with those with high PART1 expression levels. Combined with previous reports, it is hypothesized that differential expression patterns of PART1 in different types of tumor are dependent on the various tumor types. Overall, the data from the present study supports PART1, as a potential molecular marker for prognosis in patients with ESCC.

Transcriptional regulation of lncRNAs is important for lncRNA expression level in numerous types of tumor. A number of IncRNAs that have been identified to be abnormally expressed were found to be regulated by either a tumor suppressor, oncogenic pathways or other transcriptional factors, such as myc, p53 and NF- $\mathrm{B}$ (38-40). In pancreatic cancer, hypoxia-inducible factor- $1 \alpha$ induced the transcription of lncRNA-BX111 in the presence of hypoxia, which contributed to the hypoxia-induced epithelial-mesenchymal transition progression by regulating the expression level of ZEB1 (12). In addition, the E2F transcription factor was found to bind to the core promoter region of TINCR to induce the expression of TINCR in gastric carcinoma, which was verified using in vivo chromatin IP (41). To address the possible reason for the low expression level of PART1 in ESCC tissues and cells, the UCSC and JASPAR online software programs were used to predict the binding site of FOXP2, within the upstream promoter region of PART1, and was further confirmed using a reporter assay in the current study. Increasing evidence has demonstrated that FOXP2 plays important roles in cell proliferation, invasion and metastasis in a variety of different tumors $(42,43)$, which suggest that FOXP2 is associated with tumor development and progression. To preliminarily investigate the function of FOXP2 in ESCC, the low expression level of FOXP2 was verified using TCGA database, and was further confirmed using RT-qPCR in 75 ESCC tissues and matched adjacent normal tissues. Notably, the PART1 level was positively correlated with FOXP2 mRNA expression level in ESCA tissues, and FOXP2 downregulation markedly suppressed the expression level of PART1; however, FOXP2 upregulation significantly increased the expression level of PART1. These data indicated that the transcription factor, FOXP2 is a direct inducer of PART1 in ESCC, and the FOXP2/PART1 signaling axis may be a novel therapeutic target; however, the functions and molecular mechanisms involved require further investigation.

It is well known that lncRNAs widely participate in the regulation of cell proliferation, invasion and metastasis in a large number of tumors. Sun et al (37) found that PART1 downregulation repressed cell proliferation and promoted cell apoptosis in prostate cancer. Furthermore, PART1 functions as a tumor suppressor by sponging miR-190a-3p to inactivate the PTEN/AKT signaling pathway in glioma (24). To further uncover the function of PART1 in ESCC cell proliferation and invasion, PART1 siRNA knockdown and pcDNA3.1-PART1 overexpression was used to investigate the role of PART1 in cell proliferation and invasion in the ESCC cell lines. It was found that PART1 downregulation significantly promoted cell proliferation and invasion, whereas PART1 upregulation markedly suppressed cell proliferation and invasion in the ESCC cell lines. These findings suggest that PART1 exerts a tumor-suppressive role in ESCC cell lines and may be a promising therapeutic target in patients with ESCC.

Determining the regulatory role of IncRNAs as key players is important to understand the etiology of several types of tumor (44). To further investigate the regulatory 

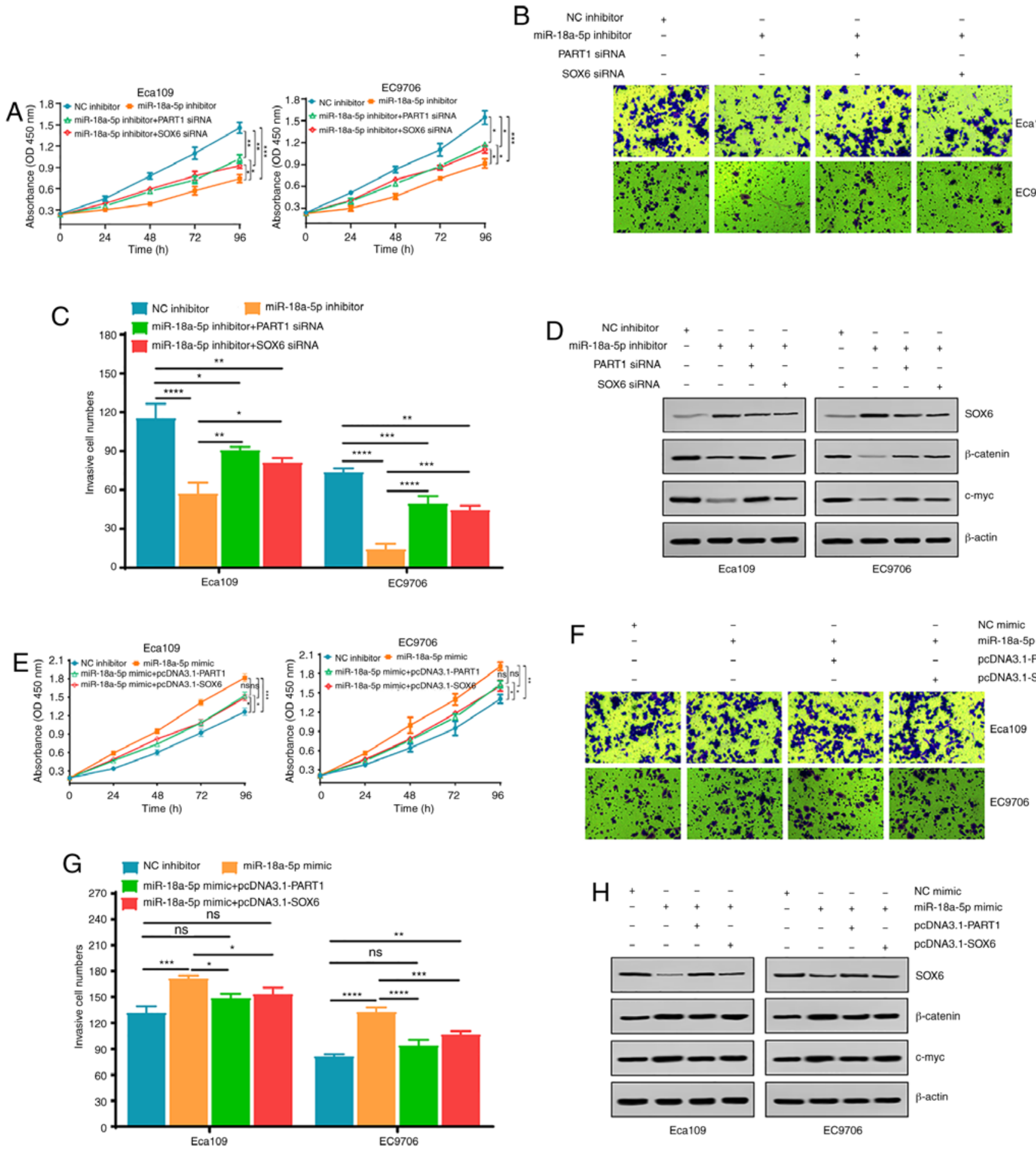

Figure 6. Biological functions exerted by miR-18a-5p are dependent on the expression levels of PART1 and SOX6 in the ESCC cell lines. (A) ESCC cell proliferation was assessed using a CCK-8 assay in the NC inhibitor, miR-18a-5p inhibitor, miR-18a-5p inhibitor plus PART1 siRNA and miR-18a-5p inhibitor plus SOX6 siRNA groups. (B) Cell invasion ability was detected using Matrigel assay in various ESCC cell lines in the different experimental groups. (C) Quantification of cell invasive in different ESCC cell lines and experimental groups. (D) Western blot analysis of SOX6, $\beta$-catenin and c-myc protein expression levels in the different experimental groups. ${ }^{*} \mathrm{P}<0.05,{ }^{* *} \mathrm{P}<0.01,{ }^{* * *} \mathrm{P}<0.001$ and ${ }^{* * * * *} \mathrm{P}<0.0001$. (E) ESCC cell proliferation was assessed using a CCK-8 assay in NC mimic, miR-18a-5p mimic, miR-18a-5p mimic plus pcDNA3.1-PART1 and miR-18a-5p mimic plus pcDNA3.1-SOX6 groups. (F) Cell invasion ability was detected using a Matrigel assay in various ESCC cell lines and the different experimental groups. (G) Quantification of cell invasive in the different ESCC cell lines and the experimental groups. (H) Western blot analysis of SOX6, $\beta$-catenin and c-myc protein expression level in the different experimental groups. ${ }^{*} \mathrm{P}<0.05,{ }^{* *} \mathrm{P}<0.01,{ }^{* * *} \mathrm{P}<0.001$ and ${ }^{* * * * *} \mathrm{P}<0.0001$; ns, not significant. CCK-8, Cell Counting Kit-8; si, small interfering; NC, negative control; miR, microRNA; ESCC, esophageal squamous cell carcinoma; PART1, prostate androgen regulated transcript 1; SOX6, SRY-box transcription factor 6. (A, C, E and G) All data were examined by one-way ANOVA and Bonferroni test.

mechanisms of PART1 in ESCC, a localization assay was performed using the lncATLAS online predictive software and RT-qPCR. It was found that PART1 was primarily localized in the cytoplasm of the ESCC cell lines, which further identified the possible function of PART1 as a ceRNA. Further investigation revealed that miR-18a-5p and 
PART1 were both enriched using an anti-Ago2 antibody, and miR-18a-5p mimic significantly increased the accumulation of PART1 in the ESCC cell lines, suggesting that miR-18a-5p and PART1 both appear in the RNA-induced silencing complex. In addition, the binding of miR-18a-5p and PART1 was confirmed using a dual-luciferase reporter assay, in which, PART1 downregulation promoted the miR-18a-5p expression level and PART1 upregulation suppressed the miR-18a-5p expression level in the ESCC cell lines. These findings suggest that PART1 could affect the expression level of miR-18a-5p using a ceRNA mechanism.

Recently, miR-18a-5p has been reported to participate in tumor progression, and may be a potential biomarker for the diagnosis and prognosis in a variety of different tumors (45-47). To further investigate the function of miR-18a-5p in ESCC development and progresion, it was found that miR-18a-5p was highly expressed in ESCC tissues and cells, and its high expression level was associated with TNM stage and lymph node metastasis in patients with ESCC, sugesting that miR-18a-5p functions as a tumor oncogene in ESCC. Further investigation revealed that SOX6 was confirmed as the downstream target gene of miR-18a-5p in the ESCC cell lines, and its expression level was negatively correlated with the miR-18a-5p expression level in 75 ESCC samples. In addition, miR-18a-5p inhibitor significantly promoted the expression level of SOX6, whereas miR-18a-5p mimic notably downregulated the expression level of SOX6. These findings suggest that SOX6 is a direct target gene of miR-18a-5p in the ESCC cell lines. To further elucidate the underlying function of miR-18a-5p in cell proliferation and invasion, CCK-8 and Matrigel assays were used and the results demonstrated that miR-18a-5p inhibitor notably blocked cell proliferation and invasion in the Eca109 and EC9706 cell lines, which was partly reversed by PART1 siRNA and SOX6 siRNA, while converse data were found when miR-18a-5p mimic was combined with pcDNA3.1-PART1 or pcDNA3.1-SOX6. These data highlight the important role of the PART1/miR-18a-5p/SOX6 signaling axis in ESCC development and progression.

Several reports have demonstrated that SOX6 downregulaion is found in ESCC, hepatocellular carcinoma and pancreatic cancer, and is assocuated with the promotion of tumor progression (48-50). Notably, SOX6 was found to be able to bind to the armadillo repeats $1-4$ of $\beta$-catenin to intercept its oncogenic activities (51), and c-myc was found to be a pivotal downstream target of $\beta$-catenin and to regulate thousands of genes (52). Based on the aforementioned research, we hypothesized that PART1 absorbs miR-18a-5p to enhance the expression level of SOX6, which further results in the inhibition of the $\beta$-catenin/c-myc signaling axis in ESCC. To further investigate the aforementioned hypothesis, the alterations of $\beta$-catenin and its downstream gene, c-myc, were investigated in this signaling axis. It was found that miR-18a-5p inhibitor triggered the upregulation of SOX6 proteins and the downregulation of $\beta$-catenin and c-myc proteins, which was also in part reversed by PART1 siRNA and SOX6 siRNA, and the inverse effect was exhibited following the utilization of miR-18a-5p mimic combined with pcDNA3.1-PART1 or pcDNA3.1-SOX6. The data in the present study suggest that the PART1/miR-18a-5p/SOX6/ $/$-catenin signaling axis may be involved in ESCC development and progression, and may be a promising therapeutic target in patients with ESCC.

In conclusion, the data in the present study revealed that PART1 was expressed at low levels in ESCC tissues and cells, and low expression levels were associated with TNM stage, lymph node metastasis and poor prognosis in patients with ESCC. PART1 was found to be a direct transcriptional target of the transcriptional factor, FOXP2, in the ESCC cell lines. PART1 overexpression and knockdown suppressed and promoted cell proliferation and invasion, respectively, in the Eca109 and EC9706 cell lines. PART1 was found to function as a ceRNA to sponge miR-18a-5p further resulting in the elevation of SOX6 expression levels, which triggered the downregulation of $\beta$-catenin and c-myc proteins, and thus, suppressed ESCC progression. The findings from the present study suggest that targeting the PART1/miR-18a-5p/SOX6/ $\beta$-catenin signaling axis may be a novel therapeutic strategy for patients with ESCC.

\section{Acknowledgements}

Not applicable.

\section{Funding}

This work was supported by Key R\&D and Promotion Projects in Henan Province (no. 182102310380), the Natural Science Foundation of Henan Province (no. 182300410377) and the Key Scientific Research Projects of Henan Higher Education Institutions (no. 17A180016).

\section{Availability of data and materials}

The data that support the findings of this study are available from the corresponding author upon reasonable request.

\section{Authors' contributions}

SY conceived the research idea and supervised this study. YZ performed the majority of experiments and interpreted the data. QZ collected the clinical samples and performed data analysis. HL, NW and XZ assisted with all the experiments and revised the manuscript. HL carried out the bioinformatics assay. YZ prepared the manuscript. All authors discussed the study, read and approved the final manuscript. All contributing authors agreed to the submission of this manuscript for publication.

\section{Ethics approval and consent to participate}

This study was reviewed and approved by the Research and Ethics Committee of the Affiliated Cancer Hospital of Zhengzhou University (Zhengzhou, China) (approval no. 2019016). The study was conducted in accordance with the International Ethical Guidelines for Biomedical Research Involving Human Subjects. All subjects provided informed consent to participate in the study.

\section{Patient consent for publication}

Not applicable. 


\section{Competing interests}

The authors declare that they have no competing interests.

\section{References}

1. Jemal A, Bray F, Center MM, Ferlay J, Ward E and Forman D: Global cancer statistics. CA Cancer J Clin 61: 69-90, 2011.

2. Abnet CC, Arnold M and Wei WQ: Epidemiology of esophageal squamous cell carcinoma. Gastroenterology 154: 360-373, 2018.

3. Yang H, Liu H, Chen Y, Zhu C, Fang W, Yu Z, Mao W, Xiang J, Han Y, Chen Z, et al: Neoadjuvant chemoradiotherapy followed by surgery versus surgery alone for locally advanced squamous cell carcinoma of the esophagus (NEOCRTEC5010): A phase III multicenter, randomized, open-label clinical trial. J Clin Oncol 36: 2796-2803, 2018

4. Herskovic A, Russell W, Liptay M, Fidler MJ and Al-Sarraf M Esophageal carcinoma advances in treatment results for locally advanced disease: Review. Ann Oncol 23: 1095-1103, 2012

5. Morris KV and Mattick JS: The rise of regulatory RNA. Nat Rev Genet 15: 423-437, 2014.

6. Parasramka MA, Maji S, Matsuda A, Yan IK and Patel T: Long non-coding RNAs as novel targets for therapy in hepatocellular carcinoma. Pharmacol Ther 161: 67-78, 2016.

7. Ulitsky I and Bartel DP: LincRNAs: Genomics, evolution, and mechanisms. Cell 154: 26-46, 2013.

8. Ma L, Bajic VB and Zhang Z: On the classification of long non-coding RNAs. RNA Biol 10: 925-933, 2013.

9. Rinn JL and Chang HY: Genome regulation by long noncoding RNAs. Annu Rev Biochem 81: 145-166, 2012.

10. Ponting CP, Oliver PL and Reik W: Evolution and functions of long noncoding RNAs. Cell 136: 629-641, 2009.

11. Rinn JL, Kertesz M, Wang JK, Squazzo SL, Xu X, Brugmann SA, Goodnough LH, Helms JA, Farnham PJ, Segal E and Chang HY: Functional demarcation of active and silent chromatin domains in human HOX loci by noncoding RNAs. Cell 129: 1311-1323, 2007.

12. Deng SJ, Chen HY, Ye Z, Deng SC, Zhu S, Zeng Z, He C, Liu ML, Huang K, Zhong JX, et al: Hypoxia-induced lncRNA-BX111 promotes metastasis and progression of pancreatic cancer through regulating ZEB1 transcription. Oncogene 37: 5811-5828, 2018.

13. Lin C, Zhang S, Wang Y, Wang Y, Nice E, Guo C, Zhang E, Yu L, Li M, Liu C, et al: Functional role of a novel long noncoding RNA TTN-AS1 in esophageal squamous cell carcinoma progression and metastasis. Clin Cancer Res 24: 486-498, 2018.

14. Pandey GK, Mitra S, Subhash S, Hertwig F, Kanduri M, Mishra K, Fransson S, Ganeshram A, Mondal T, Bandaru S, et al: The risk-associated long noncoding RNA NBAT-1 controls neuroblastoma progression by regulating cell proliferation and neuronal differentiation. Cancer Cell 26: 722-737, 2014

15. Damas ND, Marcatti M, Come C, Christensen LL, Nielsen MM, Baumgartner R, Gylling HM, Maglieri G, Rundsten CF Seemann SE, et al: SNHG5 promotes colorectal cancer cell survival by counteracting STAU1-mediated mRNA destabilization. Nat Commun 7: 13875, 2016

16. Wang K, Long B, Zhou LY, Liu F, Zhou QY, Liu CY, Fan YY and Li PF: CARL lncRNA inhibits anoxia-induced mitochondrial fission and apoptosis in cardiomyocytes by impairing miR-539-dependent PHB2 downregulation. Nat Commun 5: 3596, 2014

17. Peng WX, Koirala P and Mo YY: IncRNA-mediated regulation of cell signaling in cancer. Oncogene 36: 5661-5667, 2017.

18. Schmitt AM and Chang HY: Long noncoding RNAs in cancer pathways. Cancer Cell 29: 452-463, 2016.

19. Xiao G, Li Y, Wang Y, Zhao B, Zou Z, Hou S, Jia X, Liu X, Yao Y, Wan J and Xiong H: lncRNA PRAL is closely related to clinical prognosis of multiple myeloma and the bortezomib sensitivity. Exp Cell Res 370: 254-263, 2018.

20. Qiu L, Tang Q, Li G and Chen K: Long non-coding RNAs as biomarkers and therapeutic targets: Recent insights into hepatocellular carcinoma. Life Sci 191: 273-282, 2017.

21. Lin B, White JT, Ferguson C, Bumgarner R, Friedman C, Trask B, Ellis W, Lange P, Hood L and Nelson PS: PART-1: A novel human prostate-specific, androgen-regulated gene that maps to chromosome 5q12. Cancer Res 60: 858-863, 2000.
22. Lou T, Ke K, Zhang L, Miao C and Liu Y: IncRNA PART1 facilitates the malignant progression of colorectal cancer via miR-150-5p/LRG1 axis. J Cell Biochem 121: 4271-4281, 2020.

23. Kang M, Ren M,Li Y,Fu Y, Deng M and Li C: Exosome-mediated transfer of lncRNA PART1 induces gefitinib resistance in esophageal squamous cell carcinoma via functioning as a competing endogenous RNA. J Exp Clin Cancer Res 37: 171, 2018.

24. Jin Z, Piao L, Sun G, Lv C, Jing Y and Jin R: Long non-coding RNA PART1 exerts tumor suppressive functions in glioma via sponging miR-190a-3p and inactivation of PTEN/AKT pathway. Onco Targets Ther 13: 1073-1086, 2020.

25. Xuan C, Jin M, Wang L, Xue S, An Q, Sun Q, Wang L and Gao Y: PART1 and hsa-miR-429-Mediated SHCBP1 expression is an independent predictor of poor prognosis in glioma patients. Biomed Res Int 2020: 1767056, 2020.

26. Kim JH, Hwang J, Jung JH, Lee HJ, Lee DY and Kim SH: Molecular networks of FOXP family: Dual biologic functions, interplay with other molecules and clinical implications in cancer progression. Mol Cancer 18: 180, 2019.

27. Herrero MJ and Gitton Y: The untold stories of the speech gene, the FOXP2 cancer gene. Genes Cancer 9: 11-38, 2018

28. Gascoyne DM, Spearman H, Lyne L, Puliyadi R, Perez-Alcantara M, Coulton L, Fisher SE, Croucher PI and Banham AH: The forkhead transcription factor FOXP2 is required for regulation of p21WAF1/CIP1 in 143B osteosarcoma cell growth arrest. PLoS One 10: e0128513, 2015

29. Chen Z, Li J, Tian L, Zhou C, Gao Y, Zhou F, Shi S, Feng X, Sun N, Yao R, et al: miRNA expression profile reveals a prognostic signature for esophageal squamous cell carcinoma. Cancer Lett 350: 34-42, 2014

30. Li JH, Liu S, Zhou H, Qu LH and Yang JH: StarBase v2.0: Decoding miRNA-ceRNA, miRNA-ncRNA and protein-RNA interaction networks from large-scale CLIP-Seq data. Nucleic Acids Res 42 (Database Issue): D92-D97, 2014

31. Livak KJ and Schmittgen TD: Analysis of relative gene expression data using real-time quantitative PCR and the 2(-Delta Delta C(T)) method. Methods 25: 402-408, 2001

32. Tsai MC, Manor O, Wan Y, Mosammaparast N, Wang JK, Lan F, Shi Y, Segal E and Chang HY: Long noncoding RNA as modular scaffold of histone modification complexes. Science 329: 689-693, 2010.

33. Hajjari $M$ and Salavaty A: HOTAIR: An oncogenic long non-coding RNA in different cancers. Cancer Biol Med 12: 1-9, 2015.

34. Zou ZW, Ma C, Medoro L, Chen L, Wang B, Gupta R, Liu T, Yang XZ, Chen TT, Wang RZ, et al: lncRNA ANRIL is up-regulated in nasopharyngeal carcinoma and promotes the cancer progression via increasing proliferation, reprograming cell glucose metabolism and inducing side-population stem-like cancer cells. Oncotarget 7: 61741-61754, 2016.

35. Hua L, Wang CY, Yao KH, Chen JT, Zhang JJ and Ma WL: High expression of long non-coding RNA ANRIL is associated with poor prognosis in hepatocellular carcinoma. Int J Clin Exp Pathol 8: 3076-3082, 2015.

36. Li M, Zhang W, Zhang S, Wang C and Lin Y: PART1 expression is associated with poor prognosis and tumor recurrence in stage I-III non-small cell lung cancer. J Cancer 8: 1795-1800, 2017.

37. Sun M, Geng D, Li S, Chen Z and Zhao W: lncRNA PART1 modulates toll-like receptor pathways to influence cell proliferation and apoptosis in prostate cancer cells. Biol Chem 399: 387-395, 2018.

38. Hung T, Wang Y, Lin MF, Koegel AK, Kotake Y, Grant GD, Horlings HM, Shah N, Umbricht C, Wang P, et al: Extensive and coordinated transcription of noncoding RNAs within cell-cycle promoters. Nat Genet 43: 621-629, 2011.

39. Huarte M, Guttman M, Feldser D, Garber M, Koziol MJ, Kenzelmann-Broz D, Khalil AM, Zuk O, Amit I, Rabani M, et al: A large intergenic noncoding RNA induced by p53 mediates global gene repression in the p53 response. Cell 142: 409-419, 2010.

40. Guttman M, Amit I, Garber M, French C, Lin MF, Feldser D, Huarte M, Zuk O, Carey BW, Cassady JP, et al: Chromatin signature reveals over a thousand highly conserved large non-coding RNAs in mammals. Nature 458: 223-227, 2009.

41. Xu TP, Wang YF, Xiong WL, Ma P, Wang WY, Chen WM, Huang MD, Xia R, Wang R, Zhang EB, et al: E2F1 induces TINCR transcriptional activity and accelerates gastric cancer progression via activation of TINCR/STAU1/CDKN2B signaling axis. Cell Death Dis 8: e2837, 2017. 
42. Chen MT, Sun HF, Li LD, Zhao Y, Yang LP, Gao SP and Jin W: Downregulation of FOXP2 promotes breast cancer migration and invasion through TGF//SMAD signaling pathway. Oncol Lett 15: 8582-8588, 2018.

43. Wu J, Liu P, Tang H, Shuang Z, Qiu Q, Zhang L, Song C, Liu L, $\mathrm{Xie} \mathrm{X}$ and Xiao X: FOXP2 promotes tumor proliferation and metastasis by Targeting GRP78 in triple-negative breast cancer. Curr Cancer Drug Targets 18: 382-389, 2018.

44. Wapinski $\mathrm{O}$ and Chang HY: Long noncoding RNAs and human disease. Trends Cell Biol 21: 354-361, 2011.

45. Wang H, Wei X, Wu B, Su J, Tan W and Yang K: Tumor-educated platelet miR-34c-3p and miR-18a-5p as potential liquid biopsy biomarkers for nasopharyngeal carcinoma diagnosis. Cancer Manag Res 11: 3351-3360, 2019.

46. Li Y, He ZC, Liu Q, Zhou K, Shi Y, Yao XH, Zhang X, Kung HF, Ping YF and Bian XW: Large intergenic non-coding RNA-RoR inhibits aerobic glycolysis of glioblastoma cells via Akt pathway. J Cancer 9: 880-889, 2018.

47. Liang C, Zhang X, Wang HM, Liu XM, Zhang XJ, Zheng B, Qian GR and Ma ZL: MicroRNA-18a-5p functions as an oncogene by directly targeting IRF2 in lung cancer. Cell Death Dis 8: e2764, 2017.

48. Qin YR, Tang H, Xie F, Liu H,Zhu Y, Ai J,Chen L, Li Y, Kwong DL, $\mathrm{Fu} \mathrm{L}$ and Guan XY: Characterization of tumor-suppressive function of SOX6 in human esophageal squamous cell carcinoma. Clin Cancer Res 17: 46-55, 2011.
49. Wang J, Ding S, Duan Z, Xie Q, Zhang T, Zhang X, Wang Y, Chen X, Zhuang H and Lu F: Role of p14ARF-HDM2-p53 axis in SOX6-mediated tumor suppression. Oncogene 35: 1692-1702, 2016.

50. Jiang W, Yuan Q, Jiang Y, Huang L, Chen C, Hu G, Wan R, Wang $X$ and Yang L: Identification of Sox6 as a regulator of pancreatic cancer development. J Cell Mol Med 22: 1864-1872, 2018.

51. Iguchi H, Urashima Y, Inagaki Y, Ikeda Y, Okamura M, Tanaka T, Uchida A, Yamamoto TT, Kodama T and Sakai J: SOX6 suppresses cyclin D1 promoter activity by interacting with beta-catenin and histone deacetylase 1 , and its down-regulation induces pancreatic beta-cell proliferation. J Biol Chem 282: 19052-19061, 2007.

52. Kim J, Woo AJ, Chu J, Snow JW, Fujiwara Y, Kim CG, Cantor AB and Orkin SH: A Myc network accounts for similarities between embryonic stem and cancer cell transcription programs. Cell 143: 313-324, 2010.

This work is licensed under a Creative Commons Attribution-NonCommercial-NoDerivatives 4.0 International (CC BY-NC-ND 4.0) License. 